\title{
On the Hopf conjecture with symmetry
}

\author{
LEE KENNARD
}

The Hopf conjecture states that an even-dimensional, positively curved Riemannian manifold has positive Euler characteristic. We prove this conjecture under the additional assumption that a torus acts by isometries and has dimension bounded from below by a logarithmic function of the manifold dimension. The main new tool is the action of the Steenrod algebra on cohomology.

53C20; 55S10

Positively curved spaces have been of interest since the beginning of global Riemannian geometry. Unfortunately, there are few known examples (see Ziller [20] for a survey and Petersen and Wilhelm [12], Dearricott [7], and Grove, Verdiani and Ziller [9] for recent examples) and few topological obstructions to any given manifold admitting a positively curved metric. In fact, all known closed, simply connected examples in dimensions larger than 24 are spheres and projective spaces, and all known obstructions to positive curvature for closed, simply connected manifolds are already obstructions to nonnegative curvature.

One famous conjectured obstruction to positive curvature was made by Heinz Hopf in the 1930s. It states that closed, even-dimensional manifolds admitting positive sectional curvature have positive Euler characteristic. This conjecture holds in dimensions two and four by the theorems of Gauss and Bonnet or Bonnet and Myers (see Bishop and Goldberg [4] or Chern [5]), but it remains open in higher dimensions.

In the 1990s, Karsten Grove proposed a research program to address our lack of knowledge in this subject. The idea is to study positively curved metrics with large isometry groups. This approach has proven to be quite fruitful (see Wilking [19] or Grove [8] for surveys). Our main result falls into this category:

Theorem A Let $M^{n}$ be a connected, closed Riemannian manifold with positive sectional curvature. If $n \equiv 0 \bmod 4$ and $M$ admits an effective, isometric $T^{r}$-action with $r \geq 2 \log _{2} n-2$, then $\chi(M)>0$.

Previous results showed that $\chi\left(M^{n}\right)>0$ under the assumption of a linear bound on $r$. For example, a positively curved $n$-manifold with an isometric $T^{r}$-action has positive 
Euler characteristic if $n$ is even and $r \geq n / 8$ or if $n \equiv 0 \bmod 4$ and $r \geq n / 10$ (see Rong and $\mathrm{Su}[14]$ and $\mathrm{Su}$ and Wang [16]).

Theorem A easily implies similar results where the assumption on the symmetry rank, ie, $\operatorname{rank}(\operatorname{Isom}(M))$, is replaced by one where the symmetry degree, ie, $\operatorname{dim}(\operatorname{Isom}(M))$, is large or the cohomogeneity, ie, $\operatorname{dim}(M / \operatorname{Isom}(M))$, is small (see Section 6).

A key tool is Wilking's connectedness theorem (see [17]), which has proven to be fundamental in the study of positively curved manifolds with symmetry. The theorem relates the topology of a closed, positively curved manifold with that of its totally geodesic submanifolds of small codimension. Since fixed-point sets of isometries are totally geodesic, this becomes a powerful tool in the presence of symmetry.

Part of the utility of the connectedness theorem is to allow proofs by induction over the dimension of the manifold. Another important implication is a certain periodicity in cohomology. By using the action of the Steenrod algebra on cohomology, we refine this periodicity in some cases. For example, we prove:

Theorem B (Periodicity Theorem) Let $M^{n}$ be a closed, one-connected, positively curved manifold that contains a pair of totally geodesic, transversely intersecting submanifolds of codimensions $k_{1} \leq k_{2}$. If $k_{1}+3 k_{2} \leq n$, then $H^{*}(M ; \mathbb{Q})$ is $\operatorname{gcd}\left(4, k_{1}, k_{2}\right)$-periodic.

It follows from [17] that, under these assumptions, $H^{*}(M ; \mathbb{Q})$ is $\operatorname{gcd}\left(k_{1}, k_{2}\right)$-periodic. For a connected, closed, orientable $n$-manifold $M$ and a coefficient ring $R$, we say that $H^{*}(M ; R)$ is $k$-periodic if there exists $x \in H^{k}(M ; R)$ such that the map $H^{i}(M ; R) \rightarrow H^{i+k}(M ; R)$ induced by multiplication by $x$ is surjective for $0 \leq i<$ $n-k$ and injective for $0<i \leq n-k$. In particular, there is a surjective map of $R \cong H^{0}(M ; R)$ onto $H^{k}(M ; R)$.

To illustrate the strength of the conclusion of Theorem B, we observe the following:

- If $\operatorname{gcd}\left(4, k_{1}, k_{2}\right)=1$, then $M$ is a rational homology sphere.

- If $\operatorname{gcd}\left(4, k_{1}, k_{2}\right)=2$, then $M$ has the rational cohomology of $\mathbb{S}^{n}$ or $\mathbb{C P}^{n / 2}$.

- If $\operatorname{gcd}\left(4, k_{1}, k_{2}\right)=4$ and $n \neq \equiv 2 \bmod 4$, then $M$ has the rational cohomology ring of $\mathbb{S}^{n}, \mathbb{C P}^{n / 2}, \mathbb{H} \mathbb{P}^{n / 4}$, or $\mathbb{S}^{3} \times \mathbb{H} \mathbb{P}^{(n-3) / 4}$.

When $\operatorname{gcd}\left(4, k_{1}, k_{2}\right)=4$ and $n \equiv 2 \bmod 4$, the rational cohomology rings of $\mathbb{S}^{n}$, $\mathbb{C} \mathbb{P}^{n / 2}, \mathbb{S}^{2} \times \mathbb{H}^{(n-2) / 4}$ and

$$
M^{6}=\left(\mathbb{S}^{2} \times \mathbb{S}^{4}\right) \#\left(\mathbb{S}^{3} \times \mathbb{S}^{3}\right) \# \cdots \#\left(\mathbb{S}^{3} \times \mathbb{S}^{3}\right)
$$


are 4-periodic, but we do not know whether other examples exist in dimensions above six. This uncertainty is what prevents us from proving Theorem $\mathrm{A}$ in all even dimensions (see Section 6).

The main step in the proof of Theorem B is the following topological result:

Theorem $\mathbf{C}$ If $M^{n}$ is a closed, one-connected manifold such that $H^{*}(M ; \mathbb{Z})$ is $k$-periodic with $3 k \leq n$, then $H^{*}(M ; \mathbb{Q})$ is $\operatorname{gcd}(4, k)$-periodic.

To prove Theorem $\mathrm{C}$, we note that the assumption implies the same periodicity with coefficients in $\mathbb{Z}_{p}$. We then use the action of the Steenrod algebra for $p=2$ and $p=3$ to improve these periodicity statements with coefficients in $\mathbb{Z}_{p}$. When combined, this information implies $\operatorname{gcd}(4, k)$-periodicity with coefficients in $\mathbb{Q}$. See Proposition 1.3 and Proposition 2.1 for more general periodicity statements with coefficients in $\mathbb{Z}_{p}$, which together can be viewed as a generalization of Adem's theorem on singly generated cohomology rings (see [2]).

With the periodicity theorem in hand, we briefly explain some of the tools that go into the proof of Theorem A. The starting point is a theorem of Conner, which states that the Euler characteristic satisfies $\chi(M)=\chi\left(M^{T}\right)$, where $M^{T}$ is the fixed-point set of the torus action. Since $M$ is even-dimensional with positive curvature, $M^{T}$ is nonempty by a theorem of Berger. Writing $\chi\left(M^{T}\right)=\sum \chi(F)$ where the sum runs over components $F$ of $M^{T}$, we see that it suffices to show $\chi(F)>0$ for all $F$. In fact, we prove that every $F$ has vanishing odd Betti numbers. An important tool is another theorem of Conner, which states that, if $P$ is a manifold on which $T$ acts, then $\mathrm{b}_{\text {odd }}\left(P^{T}\right) \leq \mathrm{b}_{\text {odd }}(P)$, where $\mathrm{b}_{\text {odd }}$ denotes the sum of the odd Betti numbers. The strategy is to find a submanifold $P$ on which $T$ acts such that $\mathrm{b}_{\text {odd }}(P)=0$ and such that $F$ is a component of $P^{T}$.

In order to find such a submanifold $P$, we investigate the web of fixed-point sets of $H \subseteq T$, where $H$ ranges over subgroups of involutions. These fixed-point sets are totally geodesic submanifolds on which $T$ acts, so, under the right conditions, we can induct over dimension. In addition, studying fixed-point sets of involutions has the advantage that we can easily control the intersection data by studying the isotropy representation at a fixed point of $T$.

In order to apply the periodicity theorem, we must find a transverse intersection in this web. To strip away complication while preserving the required codimension, symmetry, and intersection data, we define an abstract graph $\Gamma$ where the vertices correspond to involutions whose fixed-point sets satisfy certain codimension and symmetry conditions. 
An edge exists between two involutions if the intersection of the corresponding fixedpoint sets is not transverse. We then break up the proof into several parts, corresponding to the structure of $\Gamma$.

Here is a short description of the individual sections. In Sections 1 and 2, we prove the mod 2 and mod $p$ generalizations, respectively, of Adem's theorem on singly generated cohomology rings. In Section 3, we bring together these results to prove Theorem C. In Section 4, we prove Theorem B, and in Section 5, we prove Theorem A. Finally, in Section 6, we derive a corollary of Theorem A, state a periodicity conjecture that would generalize Adams' theorem on singly generated cohomology rings, and explain how a proof of this conjecture would imply that Theorem A holds in all even dimensions.

\section{Acknowledgements}

This work is part of the author's PhD thesis. The author would like to thank his advisor, Wolfgang Ziller, for his encouragement and numerous suggestions along the way. The author would also like to thank Anand Dessai and Jason DeVito for helpful comments, as well as the referee for providing multiple suggestions for improvement. The author is partially supported by National Science Foundation grant DMS-1045292.

\section{Periodicity with coefficients in $\mathbb{Z}_{2}$}

We begin by stating the definition of periodicity:

Definition 1.1 For a topological space $M$, a ring $R$, and an integer $c$, we say that $x \in H^{k}(M ; R)$ induces periodicity in $H^{*}(M ; R)$ up to degree $c$ if $M$ is connected and the maps $H^{i}(M ; R) \rightarrow H^{i+k}(M ; R)$ given by multiplication by $x$ are surjective for $0 \leq i<c-k$ and injective for $0<i \leq c-k$.

When such an $x \in H^{k}(M ; R)$ exists, we say that $H^{*}(M ; R)$ is $k$-periodic up to degree $c$. If, in addition, $M$ is a closed, orientable manifold with $\operatorname{dim}(M)=c$, we say that $H^{*}(M ; R)$ is $k$-periodic.

While we could define periodic cohomology for spaces that are not connected, we will not require it. Moreover, we wish to emphasize that throughout this paper, spaces with periodic cohomology in this sense are connected. In particular, we can immediately conclude from the definition that

$$
\operatorname{dim}_{R} H^{i k}(M ; R) \leq \operatorname{dim}_{R} H^{0}(M ; R)=1
$$


and that $x^{i}$ generates $H^{i k}(M ; R)$ for $0<i<c / k$ when $R$ is a field.

As another immediate application of the definition, when $M^{n}$ is a closed, simply connected manifold with $k$-periodic $\mathbb{Z}_{p}$-cohomology ring, $H^{1+i k}\left(M ; \mathbb{Z}_{p}\right)=0$ and $H^{n-1-i k}\left(M ; \mathbb{Z}_{p}\right)=0$ for all $i$ with $0<1+i k<n$.

We remark that $H^{*}(M ; R)$ is trivially $k$-periodic up to degree $c$ when $k \geq c$. By a slight abuse of notation, we also say that $H^{*}(M ; R)$ is $k$-periodic if $2 k \leq c$ and $H^{i}(M ; R)=0$ for $0<i<c$. One thinks of 0 as the element inducing periodicity. This convention simplifies the discussion.

We start with a general lemma about periodicity:

Lemma 1.2 Let $R$ be a field. If $x \in H^{k}(M ; R)$ is a nonzero element inducing periodicity up to degree $c$ with $2 k \leq c$, and if $x^{r}=y z$ for some $1 \leq r \leq c / k$ with $\operatorname{deg}(y) \not \equiv 0 \bmod k$, then $y$ also induces periodicity. In particular, if $x=y z$ with $0<\operatorname{deg}(y)<k$, then $y$ induces periodicity.

The way in which we will use this lemma is to take an element $x$ of minimal degree that induces periodicity, and to conclude that the only factorizations of $x^{r}$ are those of the form $\left(a x^{s}\right)\left(b x^{t}\right)$ where $a, b \in R$ are multiplicative inverses and $r=s+t$.

Proof Use periodicity to write $y=y^{\prime} y^{\prime \prime}$ and $z=z^{\prime} z^{\prime \prime}$ where $y^{\prime \prime}$ and $z^{\prime \prime}$ are powers of $x, 0<\operatorname{deg}\left(y^{\prime}\right)<l$, and $0<\operatorname{deg}\left(z^{\prime}\right)<l$. Since $x$ generates $H^{k}(M ; R)$, it follows that $y^{\prime} z^{\prime}=a x$ for some multiple $a \in R$. If $a=0$, then $x^{r}=(a x) y^{\prime \prime} z^{\prime \prime}=0$, a contradiction to periodicity and the assumption that $x \neq 0$. Supposing therefore that $a \neq 0$, we may multiply by $a^{-1}$ to assume without loss of generality that $x=y^{\prime} z^{\prime}$. Since $y^{\prime \prime}$ is a power of $x$, it is easy to see that it induces periodicity up to degree $c$, so it suffices to show that $y^{\prime}$ induces periodicity up to degree $c$. Let $k^{\prime}=\operatorname{deg}\left(y^{\prime}\right)$.

Since multiplication by $x$ induces an injection $H^{i}(M ; R) \rightarrow H^{i+k}(M ; R)$ for $0<i \leq$ $c-k$, and since this map factors as multiplication by $y^{\prime}$ followed by multiplication by $z^{\prime}$, it follows that multiplication by $y^{\prime}$ induces an injection $H^{i}(M ; R) \rightarrow H^{i+k^{\prime}}(M ; R)$ for $0<i \leq c-k$. In addition, observe that multiplication by $y^{\prime}$ and then by $x$ induces a map

$$
H^{i-k}(M ; R) \rightarrow H^{i-k+k^{\prime}}(M ; R) \rightarrow H^{i+k^{\prime}}(M ; R)
$$

that is equal to the map

$$
H^{i-k}(M ; R) \rightarrow H^{i}(M ; R) \rightarrow H^{i+k^{\prime}}(M ; R)
$$

induced by multiplication by $x$ and then by $y^{\prime}$. Since the first composition is injective, and since the first map in the second is an isomorphism, we conclude that multiplication 
by $y^{\prime}$ induces an injection $H^{i}(M ; R) \rightarrow H^{i+k^{\prime}}(M ; R)$ for all $0<i \leq c-k^{\prime}$. The proof that multiplication by $y^{\prime}$ is surjective in all required degrees is similar.

In [2], Adem showed that, for a topological space $M$, if $H^{*}\left(M ; \mathbb{Z}_{2}\right)$ is isomorphic to $\mathbb{Z}_{2}[x]$ or $\mathbb{Z}_{2}[x] / x^{q+1}$ with $q \geq 2$, then $k=\operatorname{deg}(x)$ is a power of 2 . Observe that such a cohomology ring is $k$-periodic and that $k$ is the minimal period. We now prove the following generalization of Adem's theorem:

Proposition $1.3\left(\mathbb{Z}_{2}\right.$-periodicity theorem) Suppose $x \in H^{k}\left(M ; \mathbb{Z}_{2}\right)$ is nonzero and induces periodicity in $H^{*}\left(M ; \mathbb{Z}_{2}\right)$ up to degree $c$ with $2 k \leq c$. If $x$ has minimal degree among all such elements, then $k$ is a power of 2 .

The key tool in the proof is the existence of Steenrod squares, so we review some of their properties now. The Steenrod squares are group homomorphisms

$$
\mathrm{Sq}^{i}: H^{*}\left(M ; \mathbb{Z}_{2}\right) \rightarrow H^{*}\left(M ; \mathbb{Z}_{2}\right),
$$

which exist for all $i \geq 0$ and satisfy the following properties:

(1) If $y \in H^{j}\left(M ; \mathbb{Z}_{2}\right)$, then $\mathrm{Sq}^{i}(y) \in H^{i+j}\left(M ; \mathbb{Z}_{2}\right)$, and

- if $i=0$, then $\operatorname{Sq}^{i}(y)=y$,

- if $i=j$, then $\operatorname{Sq}^{i}(y)=y^{2}$, and

- if $i>j$, then $\operatorname{Sq}^{i}(y)=0$.

(2) (Cartan formula) If $y, z \in H^{*}\left(M ; \mathbb{Z}_{2}\right)$, then

$$
\mathrm{Sq}^{i}(y z)=\sum_{0 \leq j \leq i} \mathrm{Sq}^{j}(y) \mathrm{Sq}^{i-j}(z) .
$$

(3) (Adem relations) For $a<2 b$, one has the following relation among compositions of Steenrod squares:

$$
\mathrm{Sq}^{a} \mathrm{Sq}^{b}=\sum_{j=0}^{\lfloor a / 2\rfloor}\left(\begin{array}{c}
b-1-j \\
a-2 j
\end{array}\right) \mathrm{Sq}^{a+b-j} \mathrm{Sq}^{j} .
$$

A consequence of the Adem relations is the following: If $k$ is not a power of two, there exists a relation of the form $\mathrm{Sq}^{k}=\sum_{0<i<k} a_{i} \mathrm{Sq}^{i} \mathrm{Sq}^{k-i}$ for some constants $a_{i}$. Indeed, if $k=2^{c}+d$ for integers $c$ and $d \equiv 0 \bmod 2^{c+1}$, the Adem relation with $(a, b)=\left(2^{c}, d\right)$ has the property that the $j=0$ term on the right-hand side is $\mathrm{Sq}^{k}$, while the $j>0$ terms and the $\mathrm{Sq}^{a} \mathrm{Sq}^{b}$ on the left-hand side are of the form $\mathrm{Sq}^{i} \mathrm{Sq}^{k-i}$ for some $0<i<k$. (See Hatcher [10, page 497] for a complete, more detailed argument.)

The first application of the Steenrod squares in the presence of periodicity is to show the following: 
Lemma 1.4 Suppose $x \in H^{k}\left(M ; \mathbb{Z}_{2}\right)$ is nonzero and induces periodicity up to degree $c$ with $2 k \leq c$. If $x=\operatorname{Sq}^{i}(y)$ for some $i>0$, then $x$ factors as a product of elements of degree less than $k$.

Combined with Lemma 1.2, we conclude that if $i>0$ and if $x=\operatorname{Sq}^{i}(y) \in H^{k}\left(M ; \mathbb{Z}_{2}\right)$ is nonzero and induces periodicity up to degree $c$ with $2 k \leq c$, then there is another nonzero element $x^{\prime}$ inducing periodicity up to degree $c$ with $0<\operatorname{deg}\left(x^{\prime}\right)<k$.

Proof Let $i>0$ be maximal such that $x=\operatorname{Sq}^{i}(y)$ for some cohomology element $y$. Using the Cartan relation, we compute $x^{2}$ as follows:

$$
x^{2}=\operatorname{Sq}^{i}(y)^{2}=\operatorname{Sq}^{2 i}\left(y^{2}\right)-\sum_{j \neq i} \operatorname{Sq}^{j}(y) \operatorname{Sq}^{2 i-j}(y) .
$$

Now $\mathrm{Sq}^{j}(y)$ and $\mathrm{Sq}^{2 i-j}(y)$ commute, so the sum over $j \neq i$ is twice the sum over $j<i$. Hence $x^{2}=\operatorname{Sq}^{2 i}\left(y^{2}\right)$.

Next, $\operatorname{Sq}^{i}(y)=x \neq 0$ implies $i \leq \operatorname{deg}(y)$. Moreover, $i=\operatorname{deg}(y)$ implies that $x$ factors as $y^{2}$. Suppose then that $i<\operatorname{deg}(y)$. Since $k=i+\operatorname{deg}(y)<\operatorname{deg}\left(y^{2}\right)$, it follows from the surjectivity assumption of periodicity that $y^{2}=x y^{\prime}$ for some $y^{\prime}$ with $0<\operatorname{deg}\left(y^{\prime}\right)<\operatorname{deg}(y)$. Using periodicity again, observe that $\mathrm{Sq}^{j}(x)$ for $0 \leq j<k$ can be factored as $x x_{j}$ for some $x_{j} \in H^{j}\left(M ; \mathbb{Z}_{2}\right)$. Applying the Cartan formula again, we have

$$
x^{2}=\mathrm{Sq}^{2 i}\left(x y^{\prime}\right)=x \sum_{j \leq 2 i} x_{j} \mathrm{Sq}^{2 i-j}\left(y^{\prime}\right) .
$$

The injectivity assumption of periodicity implies we may cancel an $x$ and conclude that

$$
x=\sum_{j \leq 2 i} x_{j} \mathrm{Sq}^{2 i-j}\left(y^{\prime}\right)
$$

But periodicity also implies that $x$ is the generator of $H^{k}\left(M ; \mathbb{Z}_{2}\right) \cong \mathbb{Z}_{2}$, so we have $x=x_{j} \operatorname{Sq}^{2 i-j}\left(y^{\prime}\right)$ for some $j \leq 2 i$. Because $i$ was chosen to be maximal, we must have $j>0$, that is, we must have that $x$ factors as a product of elements of degree less than $k$.

We proceed to the proof of Proposition 1.3. Suppose $x \in H^{k}\left(M ; \mathbb{Z}_{2}\right)$ is nonzero, induces periodicity up to degree $c$ with $2 k \leq c$, and has minimal degree among all such elements. Assume $k$ is not a power of 2 . We will show that $x$ factors nontrivially or that $x=\operatorname{Sq}^{i}(y)$ for some $i>0$, which contradicts Lemmas 1.2 and 1.4. 
The first step is to evaluate an Adem relation of the form $\mathrm{Sq}^{k}=\sum_{0<i<k} a_{i} \mathrm{Sq}^{i} \mathrm{Sq}^{k-i}$ on $x$. Using the factorization $\operatorname{Sq}^{j}(x)=x x_{j}$ as above, together with the Cartan formula, we obtain

$$
x^{2}=\mathrm{Sq}^{k}(x)=\sum_{0<i<k} a_{i} \mathrm{Sq}^{i}\left(x x_{k-i}\right)=\sum_{0<i<k} a_{i} \sum_{0 \leq j \leq i} x x_{j} \mathrm{Sq}^{i-j}\left(x_{k-i}\right) .
$$

As in the proof of Lemma 1.4, we use periodicity to cancel the $x$ and conclude that $x=x_{j} \operatorname{Sq}^{i-j}\left(x_{k-i}\right)$ for some $0<i<k$ and $0 \leq j \leq i$. If $j>0$, we have proven a nontrivial factorization of $x$, and if $j=0$, we have proven that $x=\operatorname{Sq}^{i}\left(x_{k-i}\right)$ for some $i>0$. As explained at the beginning of the proof, this is a contradiction.

\section{Periodicity with coefficients in $\mathbb{Z}_{p}$}

In this section, we prove the $\mathbb{Z}_{p}$-analogue of Proposition 1.3:

Proposition $2.1\left(\mathbb{Z}_{p}\right.$-periodicity theorem) Let $p$ be an odd prime. Suppose $x \in$ $H^{k}\left(M ; \mathbb{Z}_{p}\right)$ is nonzero and induces periodicity in $H^{*}\left(M ; \mathbb{Z}_{p}\right)$ up to degree $c$ with $p k \leq c$. If $x$ has minimal degree among all such elements, then $k=2 \lambda p^{a}$ for some $a \geq 0$ and $\lambda \mid p-1$.

The proof uses Steenrod powers. These are group homomorphisms

$$
\mathrm{P}^{i}: H^{*}\left(M ; \mathbb{Z}_{p}\right) \rightarrow H^{*}\left(M ; \mathbb{Z}_{p}\right)
$$

for $i \geq 0$ that satisfy the following properties:

(1) If $y \in H^{j}\left(M ; \mathbb{Z}_{p}\right)$, then $\mathrm{P}^{i}(y) \in H^{j+2 i(p-1)}\left(M ; \mathbb{Z}_{p}\right)$, and the following hold:

- if $i=0$, then $\mathrm{P}^{i}(y)=y$,

- if $2 i=j$, then $\mathrm{P}^{i}(y)=y^{p}$, and

- if $2 i>j$, then $\mathrm{P}^{i}(y)=0$.

(2) (Cartan formula) For $y, z \in H^{*}\left(M ; \mathbb{Z}_{p}\right), \mathrm{P}^{i}(y z)=\sum_{0 \leq j \leq i} \mathrm{P}^{j}(y) \mathrm{P}^{i-j}(z)$.

(3) (Adem relations) For $a<p b$,

$$
\mathrm{P}^{a} \mathrm{P}^{b}=\sum_{j=0}^{\lfloor a / p\rfloor}(-1)^{a+j}\left(\begin{array}{c}
(p-1)(b-j)-1 \\
a-p j
\end{array}\right) \mathrm{P}^{a+b-j} \mathrm{P}^{j} .
$$

As with Steenrod squares, the Adem relations imply that $\mathrm{P}^{i}$ decomposes unless $i$ is a power of $p$. However, we will need a more refined statement about this decomposability. Let $\mathcal{A}$ be the algebra over $\mathbb{Z}_{p}$ generated by $\left\{\mathrm{P}^{i}\right\}_{i \geq 0}$ modulo the Adem relations. The following lemma does not use periodicity. 
Lemma 2.2 Assume $k=2\left(\lambda p^{a}+\mu\right)$ where $a \geq 0,0<\lambda<p$, and $\mu \equiv 0 \bmod p^{a+1}$. For all $1 \leq m \leq \lambda$, there exist $Q_{i} \in \mathcal{A}$ such that $\mathrm{P}^{k / 2}=\mathrm{P}^{m p^{a}} \circ Q_{a}+\sum_{i<a} \mathrm{P}^{p^{i}} \circ Q_{i}$.

Proof We induct over $k$. For $k=2$, we have $\lambda=1, m=1, a=0$, and $\mu=0$, so we can take $Q_{0}=\mathrm{P}^{0}$ to conclude the result. Suppose the result holds for all even $k^{\prime}<k$. Write $k=2\left(\lambda p^{a}+\mu\right)$ where $a \geq 0,0<\lambda<p$, and $\mu \equiv 0 \bmod p^{a+1}$, and let $1 \leq m \leq \lambda$. If $\mu=0$ and $m=\lambda$, then $\mathrm{P}^{k / 2}=\mathrm{P}^{m p^{a}}=\mathrm{P}^{m p^{a}} \circ \mathrm{P}^{0}$ is already of the desired form. If not, then $m p^{a}<p\left(k / 2-m p^{a}\right)$, and we have the Adem relation (see (1))

$$
c_{0} \mathrm{P}^{k / 2}=\mathrm{P}^{m p^{a}} \mathrm{P}^{k / 2-m p^{a}}-\sum_{j=1}^{m p^{a-1}} c_{j} \mathrm{P}^{k / 2-j} \mathrm{P}^{j} .
$$

For $1 \leq j \leq m p^{a-1}, k / 2-j$ is less than $k / 2$ and is not congruent to 0 modulo $p^{a}$. Hence, the induction hypothesis implies that each $\mathrm{P}^{k / 2-j}=\mathrm{P}^{(k-2 j) / 2}$ term is of the form $\sum_{i<a} \mathrm{P}^{p^{i}} Q_{i}$. It therefore suffices to prove that $c_{0} \not \equiv 0 \bmod p$.

For this, we use a theorem of Lucas, which states the following: For a prime $p$, if

$$
\alpha=\sum_{i \geq 0} \alpha_{i} p^{i} \quad \text { and } \quad \beta=\sum_{i \geq 0} \beta_{i} p^{i}
$$

are base $-p$ expansions of integers $\alpha$ and $\beta$, then binomial coefficient $\left(\begin{array}{l}\alpha \\ \beta\end{array}\right)$ satisfies the congruence $\left(\begin{array}{l}\alpha \\ \beta\end{array}\right) \equiv \prod\left(\begin{array}{l}\alpha_{i} \\ \beta_{i}\end{array}\right)$ mod $p$. Applying Lucas's theorem, we have that

$$
\left(\begin{array}{c}
(p-1)\left(k / 2-m p^{a}\right)-1 \\
m p^{a}
\end{array}\right) \equiv\left(\begin{array}{c}
(p-1)(\lambda-m) p^{a}-1 \\
m p^{a}
\end{array}\right) \equiv\left(\begin{array}{c}
p-(\lambda-m)-1 \\
m
\end{array}\right)
$$

modulo $p$. The first term is $(-1)^{m} c_{0}$, and the last term is nonzero modulo $p$ since $0<m \leq p-(\lambda-m)-1<p$, hence the proof is complete.

Despite the similarity of the statements of Propositions 1.3 and 2.1, the proof in the odd prime case is more involved. To simplify the remainder of the proof, we assume throughout the rest of the section that $x \in H^{k}\left(M ; \mathbb{Z}_{p}\right)$ is nonzero, induces periodicity up to degree $c$ with $p k \leq c$, and has minimal degree among all such elements. In particular, Lemma 1.2 implies that the only factorizations of $a x^{r}$ with $a \neq 0$ and $r \leq p$ are of the form $\left(a^{\prime} x^{r^{\prime}}\right)\left(a^{\prime \prime} x^{r^{\prime \prime}}\right)$ with $a^{\prime}, a^{\prime \prime} \in \mathbb{Z}_{p}$ and $r=r^{\prime}+r^{\prime \prime}$.

We proceed with a sequence of steps, the first of which is an analogue of Lemma 1.4:

Lemma 2.3 No nontrivial multiple of $x$ is of the form $\mathrm{P}^{i}(y)$ with $i>0$. 
Proof Without loss of generality, we may assume $x$ itself is equal to $\mathrm{P}^{i}(y)$ for some $i>0$. Set $d=\operatorname{deg}(y)$. Our first task is to write some power of $x$ as $\mathrm{P}^{i_{1}}\left(y_{1}\right)$ with $0<\operatorname{deg}\left(y_{1}\right)<d$. Because $x \neq 0$, we have $2 i \leq d$, which implies $\operatorname{deg}\left(y^{p}\right) \geq k$. Let $r$ be the integer such that $k+d>\operatorname{deg}\left(y^{r}\right) \geq k$. Lemma 1.2 implies a strict inequality here. Using periodicity, write $y^{r}=x y_{1}$ with $0<d_{1}<d$.

Next we use the expression $x=\mathrm{P}^{i}(y)$ and the Cartan relation to calculate

$$
x^{r}=\left(\mathrm{P}^{i}(y)\right)^{r}=\mathrm{P}^{r i}\left(y^{r}\right)-\sum c_{j_{1}, \ldots, j_{r}} \mathrm{P}^{j_{1}}(y) \cdots \mathrm{P}^{j_{r}}(y)
$$

where the $c_{j_{1}, \ldots, j_{r}}$ are constants and the sum runs over $j_{1} \geq \cdots \geq j_{r}$ with $j_{1}+\cdots+j_{r}=$ $r i$ and $\left(j_{1}, \ldots, j_{r}\right) \neq(i, \ldots, i)$. Observe that $j_{1}>i$, so $\mathrm{P}^{j_{1}}(y)=x z_{j_{1}}$ for some $z_{j_{1}} \in H^{2\left(j_{1}-i\right)(p-1)}\left(M ; \mathbb{Z}_{p}\right)$. Using $y^{r}=x y_{1}$, the first term on the right-hand side becomes

$$
\mathrm{P}^{r i}\left(y^{r}\right)=\mathrm{P}^{r i}\left(x y_{1}\right)=\sum_{0 \leq h \leq r i} \mathrm{P}^{h}(x) \mathrm{P}^{r i-h}\left(y_{1}\right)=x \sum_{0 \leq h \leq r i} x_{h} \mathrm{P}^{r i-h}\left(y_{1}\right)
$$

for some $x_{h} \in H^{2 h(p-1)}\left(M ; \mathbb{Z}_{p}\right)$. Combining these calculations, and using periodicity to cancel the $x$, we obtain

$$
x^{r-1}=\sum_{0 \leq h \leq r i} x_{h} \mathrm{P}^{r i-h}\left(y_{1}\right)+\sum c_{j_{1}, \ldots, j_{r}} z_{j_{1}} \mathrm{P}^{j_{2}}(y) \cdots \mathrm{P}^{j_{r}}(y) .
$$

Now $r-1<p$, so periodicity implies that $x^{r-1}$ generates $H^{(r-1) k}\left(M ; \mathbb{Z}_{p}\right)$. By Lemma 1.2 , every term of the form $z_{j_{1}} \mathrm{P}^{j_{2}}(y) \cdots \mathrm{P}^{j_{r}}(y)$ vanishes since

$$
0<\operatorname{deg}\left(\mathrm{P}^{j_{r}}(y)\right)<\operatorname{deg}\left(\mathrm{P}^{i}(y)\right)=k .
$$

Similarly, all terms of the form $x_{h} \mathrm{P}^{r i-h}\left(y_{1}\right)$ vanish unless $\mathrm{P}^{r i-h}\left(y_{1}\right)$ is a nontrivial multiple of a power of $x$. Hence without loss of generality, some power of $x$ is of the form $\mathrm{P}^{i_{1}}\left(y_{1}\right)$, as claimed.

We now show that, given an expression $x^{r_{j}}=\mathrm{P}^{i_{j}}\left(y_{j}\right)$ for some $j \geq 1$ with $0<$ $\operatorname{deg}\left(y_{j}\right)<d$, there exists another expression $x^{r_{j+1}}=\mathrm{P}^{i_{j+1}}\left(y_{j+1}\right)$ with

$$
0<\operatorname{deg}\left(y_{j+1}\right)<d .
$$

Moreover, it will be apparent that $k+\operatorname{deg}\left(y_{j+1}\right)=\operatorname{deg}\left(y_{j}\right)+m_{j} d$ for some integer $m_{j}$. First, with $y_{j}$ fixed, choose an expression of the form $x^{r_{j}}=\mathrm{P}^{i_{j}}\left(y_{j}\right)$ with minimal $r_{j}$ (equivalently, with minimal $i_{j}$ ). Next, note that $\mathrm{P}^{i_{j}}\left(y_{j}\right)=x^{r_{j}} \neq 0$ implies $p \operatorname{deg}\left(y_{j}\right) \geq r_{j} k$, which together with $p d \geq k$ implies

$$
p \operatorname{deg}\left(y_{j} y^{p-r_{j}}\right)=p \operatorname{deg}\left(y_{j}\right)+\left(p-r_{j}\right) p d \geq p k .
$$


Hence, we can choose an integer $m_{j} \leq p-r_{j}$ satisfying $k \leq \operatorname{deg}\left(y_{j}\right)+m_{j} d<k+d$. Once again, Lemma 1.2 implies both inequalities are strict. Using periodicity, we can write $y_{j} y^{m_{j}}=x y_{j+1}$ with $0<\operatorname{deg}\left(y_{j+1}\right)<d$ and $k+\operatorname{deg}\left(y_{j+1}\right)=\operatorname{deg}\left(y_{j}\right)+m_{j} d$. We now calculate

$$
x^{r_{j}+m_{j}}=\mathrm{P}^{i_{j}}\left(y_{j}\right) \mathrm{P}^{i}(y)^{m_{j}}=\mathrm{P}^{i_{j}+m_{j} i}\left(y_{j} y^{m_{j}}\right)-\sum \mathrm{P}^{h_{0}}\left(y_{j}\right) \mathrm{P}^{h_{1}}(y) \cdots \mathrm{P}^{h_{m_{j}}}(y)
$$

where the sum runs over $\left(h_{0}, \ldots, h_{m_{j}}\right) \neq\left(i_{j}, i, \ldots, i\right)$ with $h_{0}+\cdots+h_{m_{j}}=i_{j}+m_{j} i$. As when we calculated $x^{r}$ above, we are able to factor an $x$ from each term on the right-hand side and use periodicity to cancel it. Using Lemma 1.2 and the fact that $x^{r_{j}+m_{j}-1}$ generates $H^{\left(r_{j}+m_{j}-1\right) k}\left(M ; \mathbb{Z}_{p}\right)$, together with the assumption that $r_{j}$ is minimal, we conclude the existence of an expression of the form $x^{r_{j+1}}=\mathrm{P}^{i_{j+1}}\left(y_{j+1}\right)$, as claimed.

We therefore have a sequence of cohomology elements $y_{1}, y_{2}, \ldots$ with $0<\operatorname{deg}\left(y_{j}\right)<d$ and $k+\operatorname{deg}\left(y_{j+1}\right)=\operatorname{deg}\left(y_{j}\right)+m_{j} d$ for some integer $m_{j}$ for all $j \geq 1$. This cannot be. Indeed, adding the equations $k+\operatorname{deg}\left(y_{1}\right)=r d$ and $k+\operatorname{deg}\left(y_{j+1}\right)=\operatorname{deg}\left(y_{j}\right)+m_{j} d$ for $1 \leq j \leq d-1$ yields

$$
k d+\operatorname{deg}\left(y_{d}\right)=\left(r+m_{1}+\cdots+m_{d-1}\right) d,
$$

which implies that $\operatorname{deg}\left(y_{d}\right)$ is divisible by $d$. But $0<\operatorname{deg}\left(y_{d}\right)<d$, so this is a contradiction.

Lemma 2.3 implies the following:

Lemma 2.4 If $1 \leq r \leq p$ and $0<i<k /(2(p-1))$, then $P^{i}: H^{r k-2 i(p-1)}\left(M ; \mathbb{Z}_{p}\right) \rightarrow$ $H^{r k}\left(M ; \mathbb{Z}_{p}\right)$ is zero.

Proof Let $1 \leq r \leq p, 0<i<k /(2(p-1))$, and $y \in H^{r k-2 i(p-1)}\left(M ; \mathbb{Z}_{p}\right)$. We claim that $\mathrm{P}^{i}(y)=0$. The estimate on $i$ implies $\operatorname{deg}(y)=r k-2 i(p-1)>(r-1) k$, hence periodicity implies $y=x^{r-1} z$ for some $z$ with $0<\operatorname{deg}(z)<k$. Applying the Cartan formula and writing $\mathrm{P}^{i-j}\left(x^{r-1}\right)=x^{r-1} w_{j}$ for all $0 \leq j \leq i$, we have

$$
\mathrm{P}^{i}(y)=\sum_{0 \leq j \leq i} x^{r-1} w_{j} \mathrm{P}^{j}(z) .
$$

Suppose for a moment that some $w_{j} \mathrm{P}^{j}(z) \neq 0$. Since this element has degree $k$, the fact that $x$ generates $H^{k}\left(M ; \mathbb{Z}_{p}\right)$ implies that $b x=w_{j} \mathrm{P}^{j}(z)$ for some nonzero $b \in \mathbb{Z}_{p}$. If $j<i$, then $\operatorname{deg}\left(w_{j}\right)>0$, a contradiction to Lemma 1.2 , and if $j=i$, then $w_{j}=1$ and $b x=\mathrm{P}^{j}(z)$, a contradiction to Lemma 2.3. Hence every $w_{j} \mathrm{P}^{j}(z)=0$, so the expression $\mathrm{P}^{i}(y)=\sum x^{r-1} w_{j} \mathrm{P}^{j}(z)$ implies $\mathrm{P}^{i}(y)=0$. 
So far, we have established that powers of $x$ cannot decompose in certain ways. The next stage of the proof uses Lemma 2.2 to prove that, unless $k$ has the desired form, some power of $x$ decomposes in one of these forbidden ways. Lemma 2.5 is a step in this direction. After we prove it, we will conclude the proof of Proposition 2.1.

Lemma 2.5 Suppose that $k$ takes the form $2\left(\lambda p^{a}+\mu\right)$ with $a \geq 0,0<\lambda<p$, and $\mu \equiv 0 \bmod p^{a+1}$. For all $1 \leq m \leq \lambda$, there exists $y \in H^{p k-2 m p^{a}(p-1)}\left(M ; \mathbb{Z}_{p}\right)$ such that $x^{p}=\mathrm{P}^{m p^{a}}(y)$.

Proof Let $k=2\left(\lambda p^{a}+\mu\right)$ as in the assumption, and let $1 \leq m \leq \lambda$. By Lemma 2.2, there exist $Q_{i} \in \mathcal{A}$ such that

$$
\mathrm{P}^{k / 2}-\mathrm{P}^{m p^{a}} \circ Q_{a}=\sum_{i<a} \mathrm{P}^{p^{i}} \circ Q_{i}
$$

Evaluating on $x$, the left-hand side becomes $\mathrm{P}^{k / 2}(x)-\mathrm{P}^{m p^{a}}\left(Q_{a}(x)\right)$, or $x^{p}-\mathrm{P}^{m p^{a}}(y)$ where $y=Q_{a}(x)$. On the right-hand side, we get zero by Lemma 2.4 since $p^{i} \leq$ $p^{a-1}<k /(2(p-1))$. This completes the proof.

Proof of Proposition 2.1 Suppose $x \in H^{k}\left(M ; \mathbb{Z}_{p}\right)$ is nonzero, induces periodicity up to degree $c$ with $p k \leq c$ and has minimal degree among all such elements. Observe that $p>2$ implies $x^{3} \neq 0$, so $k$ is even. Let $p^{a}$ denote the smallest power of $p$ in the $p$-adic expansion of $k / 2$, and write $k=2\left(\lambda p^{a}+\mu\right)$ with $0<\lambda<p$ and $\mu \equiv 0 \bmod p^{a+1}$. We must show that $\mu=0$ and $\lambda \mid p-1$.

We first show that $\mu=0$. Suppose instead that $\mu>0$. Taking $m=1$ in Lemma 2.5, we have $x^{p}=\mathrm{P}^{p^{a}}(y)$ for some $y \in H^{*}\left(M ; \mathbb{Z}_{p}\right)$. Since $\mu>0$, we have

$$
p^{a}=\frac{2\left(p^{a}+p^{a+1}\right)}{2(p+1)} \leq \frac{2\left(\lambda p^{a}+\mu\right)}{2(p+1)}<\frac{k}{2(p-1)},
$$

so $\mathrm{P}^{p^{a}}: H^{p k-2 p^{a}(p-1)}\left(M ; \mathbb{Z}_{p}\right) \rightarrow H^{p k}\left(M ; \mathbb{Z}_{p}\right)$ is zero. Since $x \neq 0$ and hence $x^{p} \neq 0$ by periodicity, this is a contradiction.

Suppose therefore that $\mu=0$. Let $g=\operatorname{gcd}(\lambda, p-1)$, and observe that our task is to prove $g=\lambda$. We break the proof into two cases and proceed by contradiction.

Suppose first that $1=g<\lambda$. Choose integers $m$ and $l$ such that $m(p-1)=-1+l \lambda$. By adding or subtracting multiples of $\lambda$ and $p-1$ from $m$ and $l$, respectively, we may assume $0 \leq m<\lambda$. Moreover, since $1<\lambda$, we have $0<m<\lambda$, and since $l \lambda=m(p-1)+1<\lambda(p-1)+1$, we have $l \leq p-1$. 
By Lemma 2.5, there exists $y \in H^{p k-2 m p^{a}(p-1)}\left(M ; \mathbb{Z}_{p}\right)$ such that $x^{p}=\mathrm{P}^{m p^{a}}(y)$. Using periodicity, we can write $y=x^{p-l} z$ for some $z \in H^{2 p^{a}}\left(M ; \mathbb{Z}_{p}\right)$. Applying the Cartan formula and writing $\mathrm{P}^{m p^{a}-j}\left(x^{p-l}\right)=x w_{j}$ for all $0 \leq j \leq m p^{a}$, we have

$$
x^{p}=\sum_{0 \leq j \leq m p^{a}} x w_{j} \mathrm{P}^{j}(z) .
$$

Using periodicity, we can conclude that $b x^{p-1}=w_{j} \mathrm{P}^{j}(z)$ for some $0 \leq j \leq m p^{a}$ and some nonzero $b \in \mathbb{Z}_{p}$. By Lemma $1.2, k$ divides $\operatorname{deg}\left(\mathrm{P}^{j}(z)\right)=2 j(p-1)+2 p^{a}$. Since $p^{a}$ divides $k$, we conclude from this that $p^{a}$ divides $j$. On the other hand, $\operatorname{deg}(z)=2 p^{a}$ implies $\mathrm{P}^{j}(z)=0$ if $j>p^{a}$, so we must have $j=0$ or $j=p^{a}$. In both cases, we have that $b x^{p-1}$ has a factor of $z$ since $\mathrm{P}^{0}(z)=z$ and $\mathrm{P}^{p^{a}}(z)=z^{p}$. Since $1<\lambda$ implies $0<\operatorname{deg}(z)<k$, we have a contradiction to Lemma 1.2.

Suppose now that $1<g<\lambda$. By Lemma 2.5 , there exists $y \in H^{p k-2 p^{a}(p-1)}\left(M ; \mathbb{Z}_{p}\right)$ such that $x^{p}=\mathrm{P}^{p^{a}}(y)$. Using periodicity, write $y=x^{p-r} z$ for some $0 \leq r \leq p$ and some $z \in H^{r k-2 p^{a}(p-1)}\left(M ; \mathbb{Z}_{p}\right)$ such that $0 \leq \operatorname{deg}(z)<k$. In fact, $0<\operatorname{deg}(z)<k$ since $g<\lambda$. Observe that $r$ satisfies

$$
(p-r+1) k>(p-r) k+\operatorname{deg}(z)=\operatorname{deg}(y)=p k-2 p^{a}(p-1),
$$

or, after dividing by $2 g p^{a}$,

$$
\frac{r \lambda}{g}<\frac{\lambda}{g}+\frac{p-1}{g}
$$

Since $g>1$ and $\lambda<p-1$, this implies $r \lambda / g<p$. We will use this later.

From the expressions $x^{p}=\mathrm{P}^{p^{a}}(y)$ and $y=x^{p-r} z$, the Cartan formula implies

$$
x^{p}=\mathrm{P}^{p^{a}}\left(x^{p-r} z\right)=\sum_{0 \leq j \leq p^{a}} x^{p-r} w_{j} \mathrm{P}^{j}(z)
$$

where we have used periodicity to write $\mathrm{P}^{p^{a}-j}\left(x^{p-r}\right)=x^{p-r} w_{j}$ for all $0 \leq j \leq p^{a}$. Applying periodicity again, we conclude that $x^{r}=w_{j} \mathrm{P}^{j}(z)$ for some $j$. By Lemma 1.2, $k$ divides $\operatorname{deg}\left(w_{j}\right)$, so $p^{a}$ divides $j$. Since $j=0$ would imply that $x^{r}$ has a factor of $\mathrm{P}^{0}(z)=z$, another contradiction to Lemma 1.2, we conclude that $j=p^{a}, w_{j}=1$, and $x^{r}=\mathrm{P}^{p^{a}}(z)$.

Now recall that $r \lambda / g<p$, hence $\left(x^{r}\right)^{\lambda / g}=x^{r \lambda / g} \neq 0$ and generates

$$
H^{(r \lambda / g) k}\left(M ; \mathbb{Z}_{p}\right)
$$

On the other hand, we have

$$
\left(x^{r}\right)^{\lambda / g}=\left(\mathrm{P}^{p^{a}}(z)\right)^{\lambda / g}=\mathrm{P}^{(\lambda / g) p^{a}}\left(z^{\lambda / g}\right)-\sum \mathrm{P}^{i_{1}}(z) \cdots \mathrm{P}^{i_{\lambda / g}}(z)
$$


where the sum runs over nonnegative $i_{j}$ such that $i_{1}+\cdots+i_{\lambda / g}=(\lambda / g) p^{a}$ and $\left(i_{1}, \ldots, i_{\lambda / g}\right) \neq\left(p^{a}, \ldots, p^{a}\right)$. The first term on the right-hand side is zero by Lemma 1.2 since

$$
\operatorname{deg}\left(z^{\lambda / g}\right)=\frac{\lambda}{g} \operatorname{deg}(z) \equiv-2 \lambda p^{a}\left(\frac{p-1}{g}\right) \equiv 0 \bmod k
$$

and $0<\operatorname{deg}(z)<k$. As for the other terms on the right-hand side, Lemma 1.2 requires that any nonzero term $\mathrm{P}^{i_{1}}(z) \cdots \mathrm{P}^{i_{\lambda / g}}(z)$ has $i_{j}>0$ and $i_{j} \equiv 0 \bmod p^{a}$ for all $j$. But, there is no such term in the sum, so we conclude our desired contradiction.

Since neither of these cases occurs, we conclude $g=\lambda$ and hence that $\lambda \mid p-1$. This concludes the proof of Proposition 2.1.

\section{Proof of Theorem C}

In this section, we use Propositions 1.3 and 2.1 to prove Theorem $\mathrm{C}$ in the introduction. We are given a closed, connected, simply connected manifold $M^{n}$ and an element $x \in H^{k}(M ; \mathbb{Z})$ inducing periodicity with $3 k \leq n$. Note that if $x$ is a torsion element, then periodicity implies that $M$ is a rational homology sphere. Since $H^{*}(M ; \mathbb{Q})$ is then trivially $\operatorname{gcd}(4, k)$-periodic, we may assume $x$ is not a torsion element.

In particular, since multiplication by $x$ induces a surjection $\mathbb{Z} \cong H^{0}(M ; \mathbb{Z}) \rightarrow$ $H^{k}(M ; \mathbb{Z})$, we have that $H^{k}(M ; \mathbb{Z}) \cong \mathbb{Z}$ with generator $x$. Let $\rho: H^{k}(M ; \mathbb{Z}) \rightarrow$ $H^{k}\left(M ; \mathbb{Z}_{2}\right)$ be the reduction homomorphism induced by the coefficient map $\mathbb{Z} \rightarrow \mathbb{Z}_{2}$, and set $x_{2}=\rho(x)$.

Lemma 3.1 The element $x_{2} \in H^{k}\left(M ; \mathbb{Z}_{2}\right)$ induces periodicity in $H^{*}\left(M ; \mathbb{Z}_{2}\right)$.

Proof Recall that $\rho$ fits into the Bockstein sequence, which is the long exact sequence

$$
\cdots \longrightarrow H^{i}(M ; \mathbb{Z}) \stackrel{2 \cdot}{\longrightarrow} H^{i}(M ; \mathbb{Z}) \stackrel{\rho}{\longrightarrow} H^{i}\left(M ; \mathbb{Z}_{2}\right) \longrightarrow H^{i+1}(M ; \mathbb{Z}) \longrightarrow \cdots
$$

associated to the short exact sequence

$$
0 \longrightarrow \mathbb{Z} \stackrel{2 \cdot}{\longrightarrow} \mathbb{Z} \stackrel{\rho}{\longrightarrow} \mathbb{Z}_{2} \longrightarrow 0
$$

of coefficient rings. In particular, since $x$ generates $H^{k}(M ; \mathbb{Z}) \cong \mathbb{Z}$, exactness implies $\rho(x) \neq 0$. In addition, $H^{k+1}(M ; \mathbb{Z}) \cong H^{1}(M ; \mathbb{Z})=0$ by periodicity, so we have $H^{k}\left(M ; \mathbb{Z}_{2}\right) \cong \mathbb{Z}_{2}$ by the universal coefficients theorem. This proves that multiplication by $x_{2}$ induces a surjection (in fact, an isomorphism) $H^{0}\left(M ; \mathbb{Z}_{2}\right) \rightarrow H^{k}\left(M ; \mathbb{Z}_{2}\right)$.

On the other end, Poincaré duality implies $H^{n-k}\left(M ; \mathbb{Z}_{2}\right) \cong H^{k}\left(M ; \mathbb{Z}_{2}\right) \cong \mathbb{Z}_{2}$ and that the generator $y \in H^{n-k}\left(M ; \mathbb{Z}_{2}\right)$ satisfies $x_{2} y \neq 0$. In particular, multiplication by $x_{2}$ induces an injection $H^{n-k}\left(M ; \mathbb{Z}_{2}\right) \rightarrow H^{n}\left(M ; \mathbb{Z}_{2}\right)$. 
It remains to show that multiplication by $x_{2}$ induces isomorphisms $H^{i}\left(M ; \mathbb{Z}_{2}\right) \rightarrow$ $H^{i+k}\left(M ; \mathbb{Z}_{2}\right)$ for all $0<i<n-k$. The five lemma together with the Bockstein sequence above accomplishes this for $1<i<n-1$. For $i=1$, the five lemma only implies that multiplication map $H^{i}\left(M ; \mathbb{Z}_{2}\right) \rightarrow H^{i+k}\left(M ; \mathbb{Z}_{2}\right)$ is surjective, and for $i=n-1$, it only proves injectivity. On the other hand, Poincaré duality implies $0=H^{1}\left(M ; \mathbb{Z}_{2}\right) \cong H^{n-1}\left(M ; \mathbb{Z}_{2}\right)$, so both of these maps are isomorphisms. This concludes the proof that $x_{2} \in H^{k}\left(M ; \mathbb{Z}_{2}\right)$ induces periodicity.

Given that some element induces periodicity in $H^{*}\left(M ; \mathbb{Z}_{2}\right)$, we can consider an element $y \in H^{l}\left(M ; \mathbb{Z}_{2}\right)$ of minimal degree inducing periodicity in $H^{*}\left(M ; \mathbb{Z}_{2}\right)$. By Proposition 1.3, $l$ is a power of 2 . We claim that $l$ divides $k$. The proof relies on the following lemma:

Lemma 3.2 Let $D$ be the set of positive integers $d \leq k$ such that $H^{*}\left(M ; \mathbb{Z}_{2}\right)$ is $d$-periodic. If $d_{1}, d_{2} \in D$ with $d_{1}>d_{2}$, then $d_{1}-d_{2} \in D$.

Proof Choose elements $z_{1} \in H^{d_{1}}\left(M ; \mathbb{Z}_{2}\right)$ and $z_{2} \in H^{d_{2}}\left(M ; \mathbb{Z}_{2}\right)$ inducing periodicity in $H^{*}\left(M ; \mathbb{Z}_{2}\right)$. Since $z_{2}$ induces periodicity, there exists $z_{3} \in H^{d_{1}-d_{2}}\left(M ; \mathbb{Z}_{2}\right)$ such that $z_{1}=z_{2} z_{3}$. Since $z_{1}$ induces periodicity, Lemma 1.2 implies that $z_{3}$ does as well.

To see that this lemma implies $l \mid k$, observe that $k, l \in D$. By this lemma, $\operatorname{gcd}(k, l) \in D$. But $l$ is minimal, so $l=\operatorname{gcd}(k, l)$ and therefore divides $k$. Observe that periodicity implies $y^{k / l}=x_{2}$.

Next we show that $y$ comes from an integral element $\tilde{y} \in H^{l}(M ; \mathbb{Z})$ such that the map $H^{i}(M ; \mathbb{Z}) \rightarrow H^{i+l}(M ; \mathbb{Z})$ induced by multiplication by $\tilde{y}$ has finite kernel for all $0<i \leq n-l$. First observe by periodicity that $0=H^{1}\left(M ; \mathbb{Z}_{2}\right) \cong H^{1+l}\left(M ; \mathbb{Z}_{2}\right)$, so $b_{l+1}(M) \leq b_{l+1}\left(M ; \mathbb{Z}_{2}\right)=0$. Next consider the following portion

$$
H^{l}(M ; \mathbb{Z}) \rightarrow H^{l}\left(M ; \mathbb{Z}_{2}\right) \rightarrow H^{l+1}(M ; \mathbb{Z}) \rightarrow H^{l+1}(M ; \mathbb{Z}) \rightarrow H^{l+1}\left(M ; \mathbb{Z}_{2}\right)
$$

of the Bockstein sequence. We see that $H^{l+1}(M ; \mathbb{Z}) \rightarrow H^{l+1}(M ; \mathbb{Z})$ is a surjection and hence an isomorphism since $H^{l+1}(M ; \mathbb{Z})$ is finite. Using exactness again, we conclude $\rho: H^{l}(M ; \mathbb{Z}) \rightarrow H^{l}\left(M ; \mathbb{Z}_{2}\right)$ is surjective, so that we can choose some $\tilde{y} \in H^{l}(M ; \mathbb{Z})$ with $\rho(\tilde{y})=y$. Now $H^{k}(M ; \mathbb{Z})$ is generated by $x$, so $\tilde{y}^{k / l}=a x$ for some $a \in \mathbb{Z}$. Applying $\rho$ to both sides yields

$$
a x_{2}=\rho\left(\tilde{y}^{k / l}\right)=y^{k / l}=x_{2} \neq 0,
$$

hence $a \neq 0$. This proves for all $0<i \leq n-k$ that multiplication by $\tilde{y}$ induces a map $H^{i}(M ; \mathbb{Z}) \rightarrow H^{i+k}(M ; \mathbb{Z})$ with finite kernel. In degrees $n-k<i \leq n-l$, we 
conclude this property by observing that multiplication by $x$ and multiplication by $\tilde{y}$ commute.

Moving to rational coefficients, we conclude that $\bar{y} \in H^{l}(M ; \mathbb{Q})$, the image of $\tilde{y}$ under the coefficient map $H^{l}(M ; \mathbb{Z}) \rightarrow H^{l}(M ; \mathbb{Q})$, induces periodicity in $H^{*}(M ; \mathbb{Q})$.

A completely analogous argument using Proposition 2.1 with $p=3$ shows that $H^{*}(M ; \mathbb{Q})$ is $m$-periodic for some $m$ that divides $k$ and is of the form $2 \cdot 3^{s}$ or $4 \cdot 3^{s}$. Using an argument like that in Lemma 3.2 , we conclude that $H^{*}(M ; \mathbb{Q})$ is $g$-periodic where $g$ divides $\operatorname{gcd}(k, l, m)$ and hence divides $\operatorname{gcd}(4, k)$.

\section{Proof of Theorem B}

The starting point of the proof is the following theorem of Wilking:

Connectedness Theorem [17] Suppose $M^{n}$ is a closed Riemannian manifold with positive sectional curvature.

(1) If $N^{n-k}$ is a closed, embedded, totally geodesic submanifold of $M$, then $N \hookrightarrow$ $M$ is $(n-2 k+1)$-connected.

(2) If $N_{1}^{n-k_{1}}$ and $N_{2}^{n-k_{2}}$ are closed, embedded, totally geodesic submanifolds of $M$ with $k_{1} \leq k_{2}$, then $N_{1} \cap N_{2} \hookrightarrow N_{2}$ is $\left(n-k_{1}-k_{2}\right)$-connected.

Recall an inclusion $N \hookrightarrow M$ is called $h$-connected if $\pi_{i}(M, N)=0$ for all $i \leq h$. It follows from the relative Hurewicz theorem that the induced map $H_{i}(N ; \mathbb{Z}) \rightarrow$ $H_{i}(M ; \mathbb{Z})$ is an isomorphism for $i<h$ and a surjection for $i=h$. The following is a topological consequence of highly connected inclusions of closed, orientable manifolds:

Theorem 4.1 [17] Let $M^{n}$ and $N^{n-k}$ be closed, orientable manifolds. If $N \hookrightarrow M$ is $(n-k-l)$-connected with $n-k-2 l>0$, then there exists $e \in H^{k}(M ; \mathbb{Z})$ such that the maps $H^{i}(M ; \mathbb{Z}) \rightarrow H^{i+k}(M ; \mathbb{Z})$ given by $x \mapsto e x$ are surjective for $l \leq i<n-k-l$ and injective for $l<i \leq n-k-l$.

Combining these results with Theorem $\mathrm{C}$, we will prove in this section the following slightly stronger version of Theorem B.

Theorem 4.2 Let $M^{n}$ be a closed, one-connected Riemannian manifold with positive sectional curvature. Let $N_{1}^{n-k_{1}}$ and $N_{2}^{n-k_{2}}$ be closed, embedded, totally geodesic submanifolds of $M$ that intersect transversely. Suppose $k_{1} \leq k_{2}$. 
(1) If $k_{1}+3 k_{2} \leq n$, the rational cohomology rings of $M, N_{1}, N_{2}$, and $N_{1} \cap N_{2}$ are $\operatorname{gcd}\left(4, k_{1}, k_{2}\right)$-periodic.

(2) If $2 k_{1}+2 k_{2} \leq n$, the rational cohomology rings of $M, N_{1}, N_{2}$, and $N_{1} \cap N_{2}$ are $\operatorname{gcd}\left(4, k_{1}\right)$-periodic.

(3) If $3 k_{1}+k_{2} \leq n$ and if $N_{2}$ is one-connected, the rational cohomology rings of $N_{2}$ and $N_{1} \cap N_{2}$ are $\operatorname{gcd}\left(4, k_{1}\right)$-periodic.

We make two remarks. First, all three codimension assumptions imply that $N_{1}$ is one-connected and that $N_{1} \cap N_{2}$ is one-connected if $N_{2}$ is. This follows by the connectedness theorem since the bounds on the codimensions imply that the inclusions $N_{1} \hookrightarrow M$ and $N_{1} \cap N_{2} \hookrightarrow N_{2}$ induce isomorphisms of fundamental groups. Similarly the first two bounds on $k_{1}$ and $k_{2}$ imply that $N_{2}$ is one-connected, but the third condition does not.

Second, in the proof of Theorem A, we will only use the following consequence of Theorem 4.2:

Corollary 4.3 Let $M^{n}$ be a closed, connected positively curved manifold with $n \equiv$ $0 \bmod 4$. If $N_{1}^{n-k_{1}}$ and $N_{2}^{n-k_{2}}$ are closed, embedded, totally geodesic submanifolds that intersect transversely and have $2 k_{1}+2 k_{2} \leq n$, then $\mathrm{b}_{\text {odd }}(M)=0$ where $\mathrm{b}_{\text {odd }}$ denotes the sum of the odd Betti numbers.

Proof of Corollary 4.3 Let $\pi: \widetilde{M} \rightarrow M$ denote the universal Riemannian covering. The submanifolds $\pi^{-1}\left(N_{i}\right) \subseteq M$ are transversely intersecting, totally geodesic, $\left(n-k_{i}\right)$-dimensional submanifolds of the closed, simply connected, positively curved manifold $\widetilde{M}$. Since $2 k_{1}+2 k_{2} \leq n$, Theorem 4.2 implies that $H^{*}(\widetilde{M} ; \mathbb{Q})$ is 4-periodic.

Observe that 4-periodicity and Poincaré duality imply $\mathrm{b}_{\text {odd }}(\widetilde{M})=0$ since $\pi_{1}(\widetilde{M})=0$ and $n \equiv 0 \bmod 4$. Recall now that the transfer theorem implies that $H^{*}(M ; \mathbb{Q})$ is isomorphic to $H^{*}(\widetilde{M} ; \mathbb{Q})^{\pi_{1}(M)}$, the subring of invariant elements under the action of $\pi_{1}(M)$ on $H^{*}(\widetilde{M} ; \mathbb{Q})$. Since $\mathrm{b}_{\text {odd }}(\widetilde{M})=0$, it follows that $\mathrm{b}_{\text {odd }}(M)=0$.

We proceed now to the proof of Theorem 4.2. Recall that we have a closed, simply connected Riemannian manifold $M^{n}$ with positive sectional curvature. We also have totally geodesic, transversely intersecting submanifolds $N_{1}^{n-k_{1}}$ and $N_{2}^{n-k_{2}}$ with $k_{1} \leq$ $k_{2}$. As discussed above, we may assume that $N_{1}, N_{2}$ and $N_{1} \cap N_{2}$ are simply connected and therefore orientable.

Observe that we have $3 k_{1}+k_{2} \leq n$ in all three cases. By the corollary to the connectedness theorem, $H^{*}\left(N_{2} ; \mathbb{Z}\right)$ is $k_{1}$-periodic since the intersection is transverse. The bound 
on the codimensions implies $3 k_{1} \leq \operatorname{dim}\left(N_{2}\right)$, hence Theorem $\mathrm{C}$ implies $H^{*}\left(N_{2} ; \mathbb{Q}\right)$ is $g$-periodic, where $g=\operatorname{gcd}\left(4, k_{1}\right)$. Since $N_{1} \cap N_{2} \hookrightarrow N_{2}$ is $\operatorname{dim}\left(N_{1} \cap N_{2}\right)$-connected, $H^{*}\left(N_{1} \cap N_{2} ; \mathbb{Q}\right)$ is $g$-periodic as well. This concludes the proof of the third statement.

Assume now that $2 k_{1}+2 k_{2} \leq n$. We claim that $H^{*}(M ; \mathbb{Q})$ is $g$-periodic. Let $j: N_{2} \hookrightarrow M$ be the inclusion map, and let $x^{\prime} \in H^{g}\left(N_{2} ; \mathbb{Q}\right)$ be an element inducing periodicity in $H^{*}\left(N_{2} ; \mathbb{Q}\right)$. Because $n-2 k_{2} \geq 2 k_{1}>g$, the connectedness theorem implies $j^{*}: H^{g}(M ; \mathbb{Q}) \rightarrow H^{g}\left(N_{2} ; \mathbb{Q}\right)$ is an isomorphism. Let $x \in H^{g}(M ; \mathbb{Q})$ satisfy $j^{*}(x)=x^{\prime}$. We will show that $x$ induces $g$-periodicity in $H^{*}(M ; \mathbb{Q})$.

First, we claim that it suffices to show that $x$ induces injective maps $H^{i}(M ; \mathbb{Q}) \rightarrow$ $H^{i+g}(M ; \mathbb{Q})$ via multiplication for all $0<i \leq n-g$. Indeed, periodicity in $H^{*}\left(N_{2} ; \mathbb{Q}\right)$ implies that $x^{\prime}$ generates $H^{g}\left(N_{2} ; \mathbb{Q}\right)$, hence the fact that $j^{*}$ is an isomorphism in degree $g$ implies that $x$ generates $H^{g}(M ; \mathbb{Q})$. In particular, multiplication by $x$ induces a surjection $H^{0}(M ; \mathbb{Q}) \rightarrow H^{g}(M ; \mathbb{Q})$. As for the surjectivity of the maps $H^{i}(M ; \mathbb{Q}) \rightarrow H^{i+g}(M ; \mathbb{Q})$ for $0<i<n-g$, note that the injectivity of these maps together with Poincaré duality would imply that

$$
b_{i} \leq b_{i+g}=b_{n-i-g} \leq b_{n-i}=b_{i}
$$

for all $0<i<n-g$, where $b_{i}=\operatorname{dim} H^{i}(M ; \mathbb{Q})$. Hence equality would hold, so the proof that $H^{*}(M ; \mathbb{Q})$ is $g$-periodic would be complete.

We proceed to proof that $x$ induces an injective map $H^{i}(M ; \mathbb{Q}) \rightarrow H^{i+g}(M ; \mathbb{Q})$ via multiplication for $0<i \leq n-g$ :

- For $i<k_{1}$, first observe that connectivity and periodicity imply that the map $H^{i}(M ; \mathbb{Q}) \rightarrow H^{i+g}\left(N_{2} ; \mathbb{Q}\right)$ given by $j^{*}$ followed by multiplication by $x^{\prime}$ is injective. Since this map is the same as multiplication by $x$ followed by $j^{*}$, we conclude that multiplication by $x$ induces an injection $H^{i}(M ; \mathbb{Q}) \rightarrow$ $H^{i+g}(M ; \mathbb{Q})$ in these degrees.

- For $k_{1} \leq i \leq n-2 k_{1}+1$, first consider that, by the connectedness theorem, the inclusion $N_{1} \hookrightarrow M$ is $\left(n-k_{1}-\left(k_{1}-1\right)\right)$-connected. Hence, there exists $e_{1} \in H^{k_{1}}(M ; \mathbb{Z})$ inducing isomorphisms $H^{i}(M ; \mathbb{Z}) \rightarrow H^{i+k_{1}}(M ; \mathbb{Z})$ via multiplication for $k_{1} \leq i \leq n-2 k_{1}$. Denote by $\bar{e}_{1}$ the image of $e_{1}$ under the natural map $H^{k_{1}}(M ; \mathbb{Z}) \rightarrow H^{k_{1}}(M ; \mathbb{Q})$, and note that $\bar{e}_{1}$ satisfies the corresponding property with rational coefficients.

Next, because $g$ divides $k_{1}$, periodicity implies $\left(x^{\prime}\right)^{k_{1} / g}$ generates $H^{k_{1}}\left(N_{2} ; \mathbb{Q}\right)$. Since $j: N_{2} \hookrightarrow M$ is $\left(n-2 k_{2}+1\right)$-connected and hence $\left(2 k_{1}+1\right)$-connected, $j^{*}: H^{k_{1}}(M ; \mathbb{Q}) \rightarrow H^{k_{1}}\left(N_{2} ; \mathbb{Q}\right)$ is an isomorphism. In particular, $x^{k_{1} / g}$ generates $H^{k_{1}}(M ; \mathbb{Q})$, so $a x^{k_{1} / g}=\bar{e}_{1}$ for some $a \in \mathbb{Q}$. 
Multiplication by $\bar{e}_{1}$ induces an injection $H^{i}(M ; \mathbb{Q}) \rightarrow H^{i+k_{1}}(M ; \mathbb{Q})$ for all $k_{1} \leq i \leq n-2 k_{1}+1$, and this map factors as multiplication by $x$ followed by multiplication by $a x^{k_{1} / g-1}$, hence multiplication by $x$ induces an injection $H^{i}(M ; \mathbb{Q}) \rightarrow H^{i+g}(M ; \mathbb{Q})$ for $k_{1} \leq i \leq n-2 k_{1}+1$.

- Finally, for $n-2 k_{1}+1<i \leq n-g$, we use the following commutative diagram

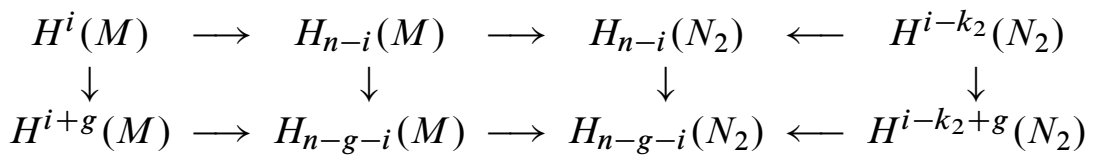

in rational cohomology where the vertical maps are cup or cap products with $x \in H^{g}(M ; \mathbb{Q})$ or $x^{\prime} \in H^{g}\left(N_{2} ; \mathbb{Q}\right)$, the horizontal maps in the middle are induced by the inclusion $j$, and the other horizontal maps are Poincare duality isomorphisms. The bounds on $i$, periodicity in $H^{*}\left(N_{2} ; \mathbb{Q}\right)$, and the connectivity of $j: N_{2} \hookrightarrow M$ imply that the rightmost vertical map is an injection and that all horizontal maps are isomorphisms. It follows that the leftmost map, namely, multiplication by $x$ from $H^{i}(M ; \mathbb{Q})$ to $H^{i+g}(M ; \mathbb{Q})$, is an injection.

This concludes the proof that $H^{*}(M ; \mathbb{Q})$ is $g$-periodic.

Next let $g^{\prime}=g$ if $k_{1}+3 k_{2}>n$ and $g^{\prime}=\operatorname{gcd}\left(4, k_{1}, k_{2}\right)$ if $k_{1}+3 k_{2} \leq n$. Our proof will be complete once we show that $M, N_{1}, N_{2}$, and $N_{1} \cap N_{2}$ are $g^{\prime}$-periodic. First, we claim that $M$ is $g^{\prime}$-periodic.

If $k_{1}+3 k_{2}>n$, then $H^{*}(M ; \mathbb{Q})$ is already $g^{\prime}$-periodic. Suppose then that $k_{1}+3 k_{2} \leq$ $n$. By the corollary to the connectedness theorem, there exists $e_{2} \in H^{k_{2}}(M ; \mathbb{Q})$ such that the maps $H^{i}(M ; \mathbb{Q}) \rightarrow H^{i+k_{2}}(M ; \mathbb{Q})$ induced by multiplication by $e_{2}$ are isomorphisms for $k_{2} \leq i \leq n-2 k_{2}$. Given that $x$ and $e_{2}$ commute, we conclude that $e_{2}$ induces periodicity in $H^{*}(M ; \mathbb{Q})$. Indeed, suppose $0 \leq i<k_{2}$. Choose $j \geq 0$ with $k_{2} \leq i+j g<k_{2}+g$. Then $i+j g \leq n-2 k_{2}$, so multiplication by $\bar{e}_{2}$ induces an isomorphism $H^{i+j g}(M ; \mathbb{Q}) \rightarrow H^{i+j g+k_{2}}(M ; \mathbb{Q})$. In addition, $i+j g+k_{2}<n$ implies that multiplication by $x^{j}$ induces an isomorphism $H^{i+k_{2}}(M ; \mathbb{Q}) \rightarrow H^{i+j g+k_{2}}(M ; \mathbb{Q})$. Hence, since multiplication by $x^{j}$ from $H^{i}(M ; \mathbb{Q})$ to $H^{i+j g}(M ; \mathbb{Q})$ is a surjection for $i=0$ and an isomorphism for $0<i<k_{1}$, it follows from commutativity that multiplication by $\bar{e}_{2}$ from $H^{i}(M ; \mathbb{Q})$ to $H^{i+k_{2}}(M ; \mathbb{Q})$ is a surjection for $i=0$ and an isomorphism for $0<i<k_{2}$. The required periodicity conditions for $n-2 k_{2}<i \leq n-k_{2}$ follow from a similar argument. Hence, we have that $H^{*}(M ; \mathbb{Q})$ is $k_{2}$-periodic and $g$-periodic. As in the proof of Lemma 3.2, it follows now that $H^{*}(M ; \mathbb{Q})$ is $\operatorname{gcd}\left(g, k_{2}\right)$-periodic and hence $g^{\prime}$-periodic.

Using this periodicity, we now conclude that the rational cohomology rings of $N_{1}, N_{2}$, and $N_{1} \cap N_{2}$ are $g^{\prime}$-periodic. First, since $4 k_{1} \leq 2 k_{1}+2 k_{2} \leq n, N_{1} \hookrightarrow M$ induces 
isomorphisms on cohomology up to half of the dimension of $N_{2}$. Using Poincaré duality, it follows from the fact that $M$ is rationally $g^{\prime}$-periodic that $N_{1}$ is too. Second, observe that $N_{2} \hookrightarrow M$ is $n-2 k_{2}+1 \geq 2 k_{1}+1$ connected. Hence $H^{*}\left(N_{2} ; \mathbb{Q}\right)$ is both $g$-periodic and $g^{\prime}$-periodic up to degree $2 k_{1}$, which is at least twice $g$. Since $N_{2}$ is rationally $g$-periodic, it follows that $N_{2}$ is rationally $g^{\prime}$-periodic by arguments similar to those above. Finally, $N_{1} \cap N_{2} \hookrightarrow N_{2}$ is $\operatorname{dim}\left(N_{1} \cap N_{2}\right)$-connected, so $N_{1} \cap N_{2}$ is clearly $g^{\prime}$-periodic as well. This concludes the proof of the Theorem 4.2.

\section{Proof of Theorem A}

Before we begin, we state two well-known theorems for easy reference:

Berger's Theorem [3] If $T$ is a torus acting by isometries on a compact, evendimensional, positively curved manifold $M$, then the fixed-point set $M^{T}$ is nonempty.

Conner's Theorem [6] If $T$ is a torus acting on a manifold $M$, then

(1) the Euler characteristic satisfies $\chi(M)=\chi\left(M^{T}\right)$, and

(2) the sum of the odd Betti numbers satisfies $\mathrm{b}_{\text {odd }}\left(M^{T}\right) \leq \mathrm{b}_{\text {odd }}(M)$.

We recall the setup of Theorem A. We are given a closed, positively curved Riemannian manifold $M^{n}$ with $n \equiv 0 \bmod 4$, and we have an effective, isometric action by a torus $T$ with $\operatorname{dim}(T) \geq 2 \log _{2}(n)-2$. By Berger's theorem, $M^{T}$ is nonempty, and, by the first part of Conner's theorem, $\chi(M)=\chi\left(M^{T}\right)$, hence it suffices to show that $\mathrm{b}_{\text {odd }}(F)=0$ for all components $F$ of $M^{T}$.

Fix a component $F$ of $M^{T}$. Our goal is to find a submanifold $P$ with $F \subseteq P \subseteq M$ and $\mathrm{b}_{\text {odd }}(P)=0$ such that $T$ acts on $P$. It would follow that $F$ is a component of $P^{T}$, hence the second part of Conner's theorem would imply $\mathrm{b}_{\text {odd }}(F)=0$.

In order to find such a submanifold $P$, the first step is to set up a sort of induction argument. To do this, we look at our situation from the point of view of $F$. We consider all closed, totally geodesic submanifolds $N$ such that

(1) $F \subseteq N \subseteq M$,

(2) $\operatorname{dim}(N) \equiv 0 \bmod 4$,

(3) the $T$-action on $M$ restricts to a $T$-action on $N$, and

(4) the kernel $\operatorname{ker}\left(\left.T\right|_{N}\right)$ of this induced action satisfies

$$
2 \log _{2}(n)-\operatorname{dim} \operatorname{ker}\left(\left.T\right|_{N}\right) \geq 2 \log _{2}(\operatorname{dim} N) .
$$


Clearly $M$ itself is one such submanifold, so the collection of submanifolds $N$ satisfying these properties is nonempty. We complete the induction setup by choosing a submanifold $N$ of minimal dimension such that these properties hold. Now that $N$ is fixed, we set $T^{\prime}=T / \operatorname{ker}\left(\left.T\right|_{N}\right)$.

Before continuing with the proof, we make a few remarks. First, observe that $T^{\prime}$ acts effectively on $N$ and that

$$
\operatorname{dim}\left(T^{\prime}\right)=\operatorname{dim}(T)-\operatorname{dim} \operatorname{ker}\left(\left.T\right|_{N}\right) \geq 2 \log _{2}(\operatorname{dim} N)-2
$$

by property (4) above. Second, observe that $F$ is a component of the fixed-point set $N^{T^{\prime}}$ since $F \subseteq N \subseteq M$. Since our goal is to show $\mathrm{b}_{\text {odd }}(F)=0$, and since we will do this by finding a submanifold $F \subseteq P \subseteq N$ on which $T^{\prime}$ acts with $\mathrm{b}_{\text {odd }}(P)=0$, we may forget about $M$ and $T$ and instead focus on $N$ and $T^{\prime}$. For this reason, it will be convenient to adopt the following notation (recall that $F, N$, and $T^{\prime}$ are fixed):

Definition 5.1 (1) For a submanifold $Q \subseteq N$, let $\operatorname{cod}(Q)$ denote the codimension of $Q$ in $N$.

(2) For a subgroup $H \subseteq T^{\prime}$, let $F(H)$ denote the component of the fixed-point set $N^{H}$ of $H$ containing $F$. If $H$ is generated by $\sigma \in T^{\prime}$ or by a collection of elements $\sigma_{1}, \ldots, \sigma_{i} \in T^{\prime}$, we will write $F(\sigma)$ or $F\left(\left\langle\sigma_{1}, \ldots, \sigma_{i}\right\rangle\right)$, respectively, in place of $F(H)$.

The first step in the proof is to apply the minimality of our choice of $N$. We remark that this is one of the two places where the logarithmic bound appears. We will refer to it frequently.

Lemma 5.2 For a nontrivial subgroup $H \subseteq T^{\prime}$ with $\operatorname{dim} F(H) \equiv 0 \bmod 4$, we have $\operatorname{dim} F(H)>(\operatorname{dim} N) / 2^{d / 2}$ where $d=\operatorname{dim} \operatorname{ker}\left(\left.T^{\prime}\right|_{F(H)}\right)$.

Proof Suppose that $\operatorname{dim} F(H) \leq(\operatorname{dim} N) / 2^{d / 2}$. Combining this estimate with the fact $N$ satisfies property (4), we have

$$
\begin{aligned}
2 \log _{2}(n)-\operatorname{dim} \operatorname{ker}\left(\left.T\right|_{F(H)}\right) & =2 \log _{2}(n)-\operatorname{dim} \operatorname{ker}\left(\left.T\right|_{N}\right)-\operatorname{dim} \operatorname{ker}\left(\left.T^{\prime}\right|_{F(H)}\right) \\
& \geq 2 \log _{2}(\operatorname{dim} N)-d \\
& \geq 2 \log _{2}(\operatorname{dim} F(H))
\end{aligned}
$$

It follows that $F(H)$ satisfies properties (1), (2), (3), and (4) above. But $\operatorname{dim} F(H)<$ $\operatorname{dim} N$ since the action of $T^{\prime}$ on $N$ is effective, so this contradicts our choice of $N$. 
We now proceed with the second part of the proof, in which we study the array of intersections of fixed-point sets of involutions in $T^{\prime}$. The strategy is to find $H \subseteq T^{\prime}$ such that $\mathrm{b}_{\text {odd }}(F(H))=0$. Since $F \subseteq F(H)$ and $T^{\prime}$ acts on $F(H)$, we set $P=F(H)$ and conclude from our remarks at the beginning of the proof that $\mathrm{b}_{\text {odd }}(F)=0$. This would complete the proof of Theorem A.

In the course finding such a submanifold $F(H) \subseteq N$, we will use Corollary 4.3 to conclude $\mathrm{b}_{\text {odd }}(F(H))=0$. This requires finding a pair of totally geodesic submanifolds of $F(H)$ that intersect transversely in $F(H)$ and have small codimension in $F(H)$. We will conclude that the codimensions are small by applying Lemma 5.2, but finding a transversely intersecting pair takes work and is the heart of the proof. To organize the required intersection, codimension, and symmetry data, we define an abstract graph that simplifies the picture while retaining this information.

Definition 5.3 Define a graph $\Gamma$ by declaring the following:

- An involution $\sigma \in T^{\prime}$ is in $\Gamma$ if $\operatorname{cod} F(\sigma) \equiv 0 \bmod 4$ and $\operatorname{dim} \operatorname{ker}\left(\left.T^{\prime}\right|_{F(\sigma)}\right) \leq 1$.

- An edge exists between distinct $\sigma, \tau \in \Gamma$ if $F(\sigma) \cap F(\tau)$ is not transverse.

We are ready to prove the existence of a submanifold $F(H) \subseteq N$ with $\mathrm{b}_{\text {odd }}(F(H))=0$. As we will see, $F(H)$ may be $N$ itself. In this case, $H$ is simply the identity subgroup. We separate the proof into five cases, according to the structure of $\Gamma$.

Lemma 5.4 (Case 1) Let $r=\operatorname{dim}\left(T^{\prime}\right)$. If $\Gamma$ does not contain $r-1$ algebraically independent involutions, then $\mathrm{b}_{\text {odd }}(N)=0$.

Proof Let $0 \leq j \leq r-2$ be maximal such that there exist $\iota_{1}, \ldots, \iota_{j} \in \Gamma$ generating a $\mathbb{Z}_{2}^{j}$. We wish to show that $\mathrm{b}_{\text {odd }}(N)=0$.

Consider the isotropy representation $\phi: T^{\prime} \hookrightarrow \mathrm{SO}\left(T_{x} N\right)$ for some $x \in F$. Choose a basis of the tangent space so that the image of the $\mathbb{Z}_{2}^{r} \subseteq T^{\prime}$ lies in a copy of $\mathbb{Z}_{2}^{m} \subseteq T^{m} \subseteq \operatorname{SO}\left(T_{x} N\right)$ where $m=\frac{1}{2} \operatorname{dim} N$. In particular, every $\sigma \in \mathbb{Z}_{2}^{r}$ has an image of the form

$$
\phi(\sigma)=\operatorname{diag}\left(\epsilon_{1} I_{2}, \epsilon_{2} I_{2}, \ldots, \epsilon_{m} I_{2}\right)
$$

for some $\epsilon_{i} \in\{1,-1\}$ where $I_{2}$ is the 2-by-2 identity matrix. Observe that $\operatorname{cod} F(\sigma)$ is the dimension of the $(-1)$-eigenspace of $\phi(\sigma)$, and that this is exactly twice the number of $\epsilon_{i}$ that equal -1 . In particular, $\operatorname{cod} F(\sigma)$ is divisible by four if and only if the number of $\epsilon_{i}=-1$ is even. Define a map $\mathbb{Z}_{2}^{r} \rightarrow \mathbb{Z}_{2}$ by composing $\sigma \mapsto \phi(\sigma)$ with the map that takes $\phi(\sigma)=\operatorname{diag}\left(\epsilon_{1} I_{2}, \ldots, \epsilon_{m} I_{2}\right)$ to $\prod_{i=1}^{m} \epsilon_{i} \in\{1,-1\}$. This map is clearly a homomorphism. Moreover, $\sigma \in \mathbb{Z}_{2}^{r}$ lies in the kernel if and only if 
$\operatorname{cod} F(\sigma) \equiv 0 \bmod 4$. Hence there exists $\mathbb{Z}_{2}^{r-1} \subseteq \mathbb{Z}_{2}^{r}$ such that every $\sigma \in \mathbb{Z}_{2}^{r-1}$ has $\operatorname{cod} F(\sigma) \equiv 0 \bmod 4$.

Since $j \leq r-2$, there exists $\iota_{j+1} \in \mathbb{Z}_{2}^{r-1} \backslash\left\langle\iota_{1}, \ldots, \iota_{j}\right\rangle$. Choosing $\iota_{j+1}$ to have minimal $\operatorname{cod} F\left(\iota_{j+1}\right)$ ensures that $\operatorname{dim} \operatorname{ker}\left(\left.T^{\prime}\right|_{F\left(\iota_{j+1}\right)}\right) \leq 2$. Indeed, $\operatorname{dim} \operatorname{ker}\left(\left.T^{\prime}\right|_{F\left(\iota_{j+1}\right)}\right) \geq 3$ would imply that a three-torus, and hence a copy of $\mathbb{Z}_{2}^{3}$, fixes $F\left(\iota_{j+1}\right)$. As argued in the previous paragraph, we could then choose a $\mathbb{Z}_{2}^{2} \subseteq \mathbb{Z}_{2}^{3}$ such that every $\sigma \in \mathbb{Z}_{2}^{2}$ has $\operatorname{cod} F(\sigma) \equiv 0 \bmod 4$. We could then choose $\sigma \in \mathbb{Z}_{2}^{2} \backslash\left\langle\iota_{j+1}\right\rangle$. Because the action of $T^{\prime}$ is effective, it would follow that $F\left(\iota_{j+1}\right) \subseteq F(\sigma) \subseteq N$ and $F\left(\iota_{j+1}\right) \subseteq F\left(\sigma \iota_{j+1}\right) \subseteq N$ with all inclusions strict. Since $\sigma$ or $\sigma \iota_{j+1}$ lies in $\mathbb{Z}_{2}^{r-1} \backslash\left\langle\iota_{1}, \ldots, \iota_{j}\right\rangle$, this would contradict the choice of $\iota_{j+1}$.

Next, because $j$ is maximal, we cannot have $\iota_{j+1} \in \Gamma$. Hence $\operatorname{cod} F\left(\iota_{j+1}\right) \equiv 0 \bmod 4$ implies that dim $\operatorname{ker}\left(\left.T^{\prime}\right|_{F\left(\iota_{j+1}\right)}\right)=2$. In particular, a copy of $\mathbb{Z}_{2}^{2} \subseteq T^{r}$ fixes $F\left(\iota_{j+1}\right)$, hence there exists $\iota \in \mathbb{Z}_{2}^{2} \backslash\left\langle\iota_{j+1}\right\rangle$. Effectivity of the $T^{\prime}$-action implies that $F\left(\iota_{j+1}\right) \subseteq$ $F(\iota) \subseteq M$ with both inclusions strict.

We claim that $F\left(\iota_{j+1}\right)$ is the transverse intersection of $F(\iota)$ and $F\left(\iota_{j+1}\right)$. Indeed, write the images of $\iota_{j+1}, \iota$, and their product as

$$
\begin{aligned}
\phi\left(\iota_{j+1}\right) & =\operatorname{diag}\left(\epsilon_{1} I_{2}, \ldots, \epsilon_{m} I_{2}\right), \\
\phi(\iota) & =\operatorname{diag}\left(\delta_{1} I_{2}, \ldots, \delta_{m} I_{2}\right),
\end{aligned}
$$

and

$$
\phi\left(\iota \iota_{j+1}\right)=\operatorname{diag}\left(\epsilon_{1} \delta_{1} I_{2}, \ldots, \epsilon_{m} \delta_{m} I_{2}\right)
$$

where $\epsilon_{i}, \delta_{i} \in\{1,-1\}$. Since $F\left(\iota_{j+1}\right) \subseteq F(\iota), \delta_{i}=-1$ implies $\epsilon_{i}=-1$. In particular, $\delta_{i}$ and $\epsilon_{i} \delta_{i}$ cannot both be -1 . Since $(-1)$ 's in the isotropy correspond to normal directions of the fixed-point set, this is precisely the statement that $F\left(\iota_{j+1}\right)$ and $F\left(\iota \iota_{j+1}\right)$ are transverse.

Finally, Lemma 5.2 and $\operatorname{dim} \operatorname{ker}\left(\left.T^{\prime}\right|_{F\left(\iota_{j+1}\right)}\right)=2$ imply that $\operatorname{dim} F\left(\iota_{j+1}\right)>(\operatorname{dim} N) / 2$, so

$$
2 \operatorname{cod} F(\iota)+2 \operatorname{cod} F\left(\iota_{j+1}\right)=2 \operatorname{cod} F\left(\iota_{j+1}\right)<\operatorname{dim} N .
$$

It follows from Corollary 4.3 that $\mathrm{b}_{\text {odd }}(N)=0$.

Lemma 5.5 (Case 2) If there exist distinct $\sigma, \tau \in \Gamma$ such that $\operatorname{dim} \operatorname{ker}\left(\left.T^{\prime}\right|_{F(\langle\sigma, \tau\rangle)}\right) \geq$ 3 , then $\mathrm{b}_{\text {odd }}(F(\tau))=0$.

Proof Let $H=\langle\sigma, \tau\rangle$. Since $\operatorname{dim} \operatorname{ker}\left(\left.T^{\prime}\right|_{F(H)}\right) \geq 3$ and $\operatorname{dim} \operatorname{ker}\left(\left.T^{\prime}\right|_{F(\tau)}\right) \leq 1$, there exists a 2-torus that acts almost effectively on $F(\tau)$ and fixes $F(H)$. Restricting our attention to the action on $F(\tau)$, we may divide by the kernel of this action to conclude that a 2-torus acts effectively on $F(\tau)$ and fixes $F(H)$. This implies the existence of 
an involution $\iota$ such that $F(H) \subseteq F(\iota) \subseteq F(\tau)$ with all inclusions strict. Since $F(H)$ is the $F$-component of the fixed-point set of the $\sigma$-action on $F(\tau)$, it follows that $F(H)$ is the transverse intersection inside $F(\tau)$ of $F\left(\left.\iota\right|_{F(\tau)}\right)$ and $F\left(\left.\iota \sigma\right|_{F(\tau)}\right)$.

Lemma 5.2 implies $\operatorname{dim} F(\tau) \geq(\operatorname{dim} N) / \sqrt{2}>\frac{2}{3} \operatorname{dim} N$ and similarly for $\operatorname{dim} F(\sigma)$. Hence,

$$
\begin{aligned}
\operatorname{cod}_{F(\tau)} F\left(\left.\iota\right|_{F(\tau)}\right)+\operatorname{cod}_{F(\tau)} F\left(\left.\iota \sigma\right|_{F(\tau)}\right) & \\
& =\operatorname{cod}_{F(\tau)} F\left(\left.\sigma\right|_{F(\tau)}\right) \leq \operatorname{cod} F(\sigma)<\frac{1}{2} \operatorname{dim} F(\tau) .
\end{aligned}
$$

Corollary 4.3 , together with the observation

$$
\operatorname{dim} F(\tau)=\operatorname{dim} N-\operatorname{cod} F(\tau) \equiv 0 \bmod 4,
$$

therefore implies $\mathrm{b}_{\text {odd }}(F(\tau))=0$.

Lemma 5.6 (Case 3) If there exist distinct $\sigma, \tau \in \Gamma$ with no edge connecting them, then $\mathrm{b}_{\text {odd }}(N)=0$ or $\mathrm{b}_{\text {odd }}(F(\tau))=0$.

Proof Let $H=\langle\sigma, \tau\rangle$. If $\operatorname{dim} \operatorname{ker}\left(\left.T^{\prime}\right|_{F(H)}\right) \geq 3$, then $\mathrm{b}_{\text {odd }}(F(\tau))=0$ by Lemma 5.5. We may assume therefore that $\operatorname{dim} \operatorname{ker}\left(\left.T^{\prime}\right|_{F(H)}\right) \leq 2$. By Lemma 5.2, $\operatorname{dim} F(H)>$ $\frac{1}{2} \operatorname{dim} N$. The assumption that no edge exists between $\sigma$ and $\tau$ means that $F(\sigma) \cap F(\tau)$ is transverse. Since

$$
2 \operatorname{cod} F(\sigma)+2 \operatorname{cod} F(\tau)=2 \operatorname{cod} F(H)<\operatorname{dim} N,
$$

Corollary 4.3 implies $\mathrm{b}_{\text {odd }}(N)=0$.

Lemma 5.7 (Case 4) If there exist distinct $\sigma, \tau \in \Gamma$ such that $\sigma \tau \notin \Gamma$, then $\mathrm{b}_{\text {odd }}(F(\tau))=0$ or $\mathrm{b}_{\text {odd }}(N)=0$.

Proof Keeping the notation from the proof of Lemma 5.4, we may permute the basis of the isotropy representation so that the images of $\sigma$ and $\tau$ take the form

and

$$
\phi(\sigma)=\operatorname{diag}(-I,-I, I, I)
$$

$$
\phi(\tau)=\operatorname{diag}(-I, I,-I, I)
$$

where the sizes of the blocks in both matrices are the even numbers $a, \operatorname{cod} F(\sigma)-a$, $\operatorname{cod} F(\tau)-a$, and $\operatorname{dim} F(\langle\sigma, \tau\rangle)$. It follows that

$\operatorname{cod} F(\sigma \tau)=\operatorname{cod} F(\sigma)+\operatorname{cod} F(\tau)-2 a \equiv \operatorname{cod} F(\sigma)+\operatorname{cod} F(\tau) \equiv 0 \bmod 4$. 
Since $\sigma \tau \notin \Gamma$, we must have $\operatorname{dim} \operatorname{ker}\left(\left.T^{\prime}\right|_{F(\sigma \tau)}\right) \geq 2$. On the other hand, the fact that $F(H) \subseteq F(\sigma \tau)$ implies

$$
\operatorname{dim} \operatorname{ker}\left(\left.T^{\prime}\right|_{F(\sigma \tau)}\right) \leq \operatorname{dim} \operatorname{ker}\left(\left.T^{\prime}\right|_{F(H)}\right),
$$

and by Lemma 5.5, we may assume that $\operatorname{dim} \operatorname{ker}\left(\left.T^{\prime}\right|_{F(H)}\right) \leq 2$, hence we have $\operatorname{dim} \operatorname{ker}\left(\left.T^{\prime}\right|_{F(\sigma \tau)}\right)=2$.

This implies the existence of an involution $\rho \in T^{\prime}$ satisfying $F(\sigma \tau) \subseteq F(\rho) \subseteq M$ with all inclusions strict, which in turn implies $F(\sigma \tau)$ is the transverse intersection in $M$ of $F(\rho)$ and $F(\rho \sigma \tau)$. Additionally, $\operatorname{dim} \operatorname{ker}\left(\left.T^{\prime}\right|_{F(\sigma \tau)}\right)=2 \operatorname{implies} \operatorname{dim} F(\sigma \tau)>$ $\frac{1}{2} \operatorname{dim} N$ by Lemma 5.2. Hence,

$$
2 \operatorname{cod} F(\rho)+2 \operatorname{cod} F(\rho \sigma \tau)=2 \operatorname{cod} F(\sigma \tau)<\operatorname{dim} N,
$$

so Corollary 4.3 implies $b_{\text {odd }}(N)=0$.

We pause before considering the last case. By the proof of Cases 3 and 4, we may assume that $\Gamma$ is a complete graph and that the set of vertices in $\Gamma$ is closed under multiplication in $T^{\prime}$. Adding the proof of Case 1, we may assume that $\Gamma$ is a complete graph on $\mathbb{Z}_{2}^{s}$ for some $s \geq \operatorname{dim}\left(T^{\prime}\right)-1$. The last case considers this possibility.

Lemma 5.8 (Case 5) Suppose $\Gamma$ is a complete graph on $\mathbb{Z}_{2}^{s}$ with $s \geq \operatorname{dim}\left(T^{\prime}\right)-1$. There exists $H \subseteq T^{\prime}$ such that $\mathrm{b}_{\text {odd }}(F(H))=0$.

Proof Set $l=\lfloor(s+1) / 2\rfloor$. We claim that there exist subgroups

$$
\Gamma \supseteq \mathbb{Z}_{2}^{s} \supseteq \mathbb{Z}_{2}^{s-1} \supseteq \cdots \supseteq \mathbb{Z}_{2}^{s-(l-1)}
$$

and involutions

$$
\rho_{i} \in \mathbb{Z}_{2}^{s-(i-1)} \backslash\left\langle\rho_{1}, \ldots, \rho_{i-1}\right\rangle
$$

for $1 \leq i \leq l$ such that the following holds for all $\rho \in \mathbb{Z}_{2}^{s-(i-1)}$ :

- $\operatorname{cod}\left(F\left(\left.\rho\right|_{R_{i-1}}\right) \subseteq R_{i-1}\right) \equiv 0 \bmod 4$, and

- $\operatorname{cod}\left(F\left(\left.\rho\right|_{R_{i-1}}\right) \subseteq R_{i-1}\right) \leq k_{i}$

where $R_{i-1}=F\left(\left\langle\rho_{1}, \ldots, \rho_{i-1}\right\rangle\right), R_{0}=M$ and $k_{i}=\operatorname{cod}\left(F\left(\left.\rho_{i}\right|_{R_{i-1}}\right) \subseteq R_{i-1}\right)=$ $\operatorname{cod}\left(R_{i} \subseteq R_{i-1}\right)$.

To see this, we first take $\mathbb{Z}_{2}^{s}$ to be the entire vertex set of $\Gamma$, and we choose $\rho_{1} \in \mathbb{Z}_{2}^{s}$ such that $k_{1}=\operatorname{cod} F\left(\rho_{1}\right)=\operatorname{cod}\left(F\left(\left.\rho_{1}\right|_{R_{0}}\right) \subseteq R_{0}\right)$ is maximal. Observe that for all $\rho \in \mathbb{Z}_{2}^{s}$,

- $\operatorname{cod}\left(F\left(\left.\rho\right|_{R_{0}}\right) \subseteq R_{0}\right)=\operatorname{cod} F(\rho) \equiv 0 \bmod 4$ because $\rho \in \Gamma$, and 
- $\operatorname{cod}\left(F\left(\left.\rho\right|_{R_{0}}\right) \subseteq R_{0}\right)=\operatorname{cod} F(\rho) \leq \operatorname{cod} F\left(\rho_{1}\right)=k_{1}$ by choice of $\rho_{1}$.

Next, suppose for some $1 \leq i<l$ that we have already chosen $\Gamma \supseteq \mathbb{Z}_{2}^{s} \supseteq \cdots \supseteq \mathbb{Z}_{2}^{s-(i-1)}$ and involutions $\rho_{1}, \ldots, \rho_{i}$ satisfying the above conditions. Keeping the notation from Lemma 5.4, we may permute the basis of $T_{x} N$ so that the isotropy images of the $\rho_{i}$ take the form

$$
\begin{aligned}
& \phi\left(\rho_{1}\right)=\operatorname{diag}(-I, I, \ldots, I, I), \\
& \phi\left(\rho_{2}\right)=\operatorname{diag}(*,-I, \ldots, I, I),
\end{aligned}
$$

and

$$
\phi\left(\rho_{i}\right)=\operatorname{diag}(*, *, \ldots,-I, I)
$$

where the blocks have size $k_{1}, k_{2}, \ldots, k_{i}, \operatorname{dim}\left(R_{i}\right)$. We define a homomorphism $\mathbb{Z}_{2}^{s-(i-1)} \rightarrow \mathbb{Z}_{2}$ by composing $\phi$ with the map that takes $\phi(\rho)=\operatorname{diag}\left(\epsilon_{1} I_{2}, \ldots, \epsilon_{m} I_{2}\right)$ to $\prod_{i>k / 2} \epsilon_{i}$ where $k=k_{1}+\cdots+k_{i}$. Observe that the product of the $\epsilon_{i}$ is over indices $i$ that correspond to tangent directions of $F\left(\left\langle\rho_{1}, \ldots, \rho_{i}\right\rangle\right)=R_{i}$. In particular, $\rho \in$ $\mathbb{Z}_{2}^{s-(i-1)}$ lies in the kernel if and only if $\operatorname{cod}\left(F\left(\left.\rho\right|_{R_{i}}\right) \subseteq R_{i}\right) \equiv 0 \bmod 4$. Choose a copy of $\mathbb{Z}_{2}^{s-i} \subseteq \mathbb{Z}_{2}^{s-(i-1)}$ that lies inside this kernel, then choose $\rho_{i+1} \in \mathbb{Z}_{2}^{s-i} \backslash\left\langle\rho_{1}, \ldots, \rho_{i}\right\rangle$ such that $\operatorname{cod}\left(F\left(\left.\rho_{i+1}\right|_{R_{i}}\right) \subseteq R_{i}\right)$ is maximal. Observe that such a choice exists since $i<l=\lfloor(s+1) / 2\rfloor$. In addition, observe that, for all $\rho \in \mathbb{Z}_{2}^{s-i}$,

- $\operatorname{cod}\left(F\left(\left.\rho\right|_{R_{i}}\right) \subseteq R_{i}\right) \equiv 0 \bmod 4$ by our choice of $\mathbb{Z}_{2}^{s-i}$, and

- $\operatorname{cod}\left(F\left(\left.\rho\right|_{R_{i}}\right) \subseteq R_{i}\right) \leq \operatorname{cod}\left(F\left(\left.\rho_{i+1}\right|_{R_{i}}\right) \subseteq R_{i}\right)=k_{i+1}$ by our choice of $\rho_{i+1}$.

This completes the proof of the claim.

We now claim that our choices imply the following:

(1) $\operatorname{dim}\left(R_{h}\right) \equiv 0 \bmod 4$ for all $h$,

(2) $k_{h} \geq 2 k_{h+1}$ for all $h$, and

(3) $k_{l}=0$.

The first claim follows by observing that $\operatorname{dim}\left(R_{h}\right)=(\operatorname{dim} N)-\left(k_{1}+\cdots+k_{h}\right)$ by definition and that $k_{i} \equiv 0 \bmod 4$ for all $i$.

To prove the second claim, fix $h \geq 1$. Observe that

$$
\rho_{h} \in \mathbb{Z}_{2}^{s-(h-1)} \quad \text { and } \quad \rho_{h+1} \in \mathbb{Z}_{2}^{s-h} \subseteq \mathbb{Z}_{2}^{s-(h-1)},
$$

so $\rho_{h} \rho_{h+1} \in \mathbb{Z}_{2}^{s-(h-1)}$ as well. By our choice of $\rho_{h}$, we have both of the following:

and

$$
\begin{aligned}
& \operatorname{cod}\left(F\left(\left.\rho_{h+1}\right|_{R_{h-1}}\right)\right.\left.\subseteq R_{h-1}\right) \leq k_{h}, \\
& \operatorname{cod}\left(F\left(\left.\rho_{h} \rho_{h+1}\right|_{R_{h-1}}\right) \subseteq R_{h-1}\right) \leq k_{h} .
\end{aligned}
$$


To derive the second property from these bounds, we recall that our choice of basis for $T_{x} M$ implies that

and

$$
\begin{aligned}
\phi\left(\rho_{h}\right) & =\operatorname{diag}(*, \ldots, *,-I, I, I), \\
\phi\left(\rho_{h+1}\right) & =\operatorname{diag}(*, \ldots, *, A,-I, I)
\end{aligned}
$$

for some $A$ where the blocks are of size $k_{1}, \ldots, k_{h}, k_{h+1}, \operatorname{dim}\left(R_{h+1}\right)$. Write $A=$ $\operatorname{diag}(-I, I)$ where the blocks have size, say, $a$ and $k_{h}-a$. Then we have

$$
\operatorname{cod}\left(F\left(\left.\rho_{h+1}\right|_{R_{h-1}}\right) \subseteq R_{h-1}\right)=a+k_{h+1},
$$

and

$$
\operatorname{cod}\left(F\left(\left.\rho_{h} \rho_{h+1}\right|_{R_{h-1}}\right) \subseteq R_{h-1}\right)=\left(k_{h}-a\right)+k_{h+1} .
$$

Substituting these expressions into the bounds above on $k_{h}$ and adding the inequalities, we conclude that $2 k_{h+1} \leq k_{h}$.

Finally, we prove the third claim. The bounds on $s$ and $\operatorname{dim}\left(T^{\prime}\right) \operatorname{imply}$

$$
l=\left\lfloor\frac{s+1}{2}\right\rfloor \geq\left\lfloor\frac{\operatorname{dim}\left(T^{\prime}\right)}{2}\right\rfloor \geq \frac{\operatorname{dim}\left(T^{\prime}\right)-1}{2} \geq \log _{2}(n)-\frac{3}{2}
$$

By the second claim, $k_{l} \leq k_{1} / 2^{l-1}$, and by Lemma 5.2, $k_{1}<n / \sqrt{2}$. Putting together these estimates, we obtain

$$
k_{l}<\frac{n}{2^{l-1 / 2}} \leq \frac{n}{2^{\log _{2}(n)-2}}=4
$$

But $k_{l} \equiv 0 \bmod 4$ by our choice of $\rho_{l}$, so $k_{l}=0$. This concludes the proof of the three claims.

We now use these facts to find a transverse intersection. Let $0<j \leq l$ be the smallest index such that $k_{j}=0$. Recall that we have represented $\phi\left(\rho_{j}\right)$ as $\operatorname{diag}(*, \ldots, *,-I, I)$ with blocks of size $k_{1}, \ldots, k_{j-1}, k_{j}=0, \operatorname{dim}\left(R_{j}\right)$. Since $k_{j}=0$, we can write this as $\phi\left(\rho_{j}\right)=\operatorname{diag}\left(A_{1}, \ldots, A_{j-1}, I\right)$. Set $a_{i}$ equal to the dimension of the $(-1)$-eigenspace of $A_{i}$. Geometrically, $a_{i}$ is the codimension of

$$
F\left(\left.\rho_{i}\right|_{R_{i-1}}\right) \cap F\left(\left.\rho_{j}\right|_{R_{i-1}}\right) \subseteq F\left(\left.\rho_{i} \rho_{j}\right|_{R_{i-1}}\right) .
$$

By replacing $\rho_{j}$ by $\rho_{j-1} \rho_{j}$ if necessary, we can ensure that $a_{j-1} \leq k_{j-1} / 2$. Observe that this may change $a_{i}$ for $i<j-1$. Next, replace $\rho_{j}$ by $\rho_{j-2} \rho_{j}$ if necessary to ensure that $a_{j-2} \leq k_{j-2} / 2$. Observe again that the $a_{i}$ may have changed for $i<j-2$, but that $a_{j-1}$ does not. Continuing in this way, we may replace $\rho_{j}$ by $\rho \rho_{j}$ for some $\rho \in\left\langle\rho_{1}, \ldots, \rho_{j-1}\right\rangle$ to ensure that $a_{i} \leq k_{i} / 2$ for all $i<j$.

Now some of the $a_{j-1}, a_{j-2}, \ldots$ may be zero, but they cannot all be zero because the action of $T^{\prime}$ is effective and $\rho_{j} \notin\left\langle\rho_{1}, \ldots, \rho_{j-1}\right\rangle$. Let $1 \leq i \leq j-1$ denote the largest index where $a_{i}>0$. 
Observe that $a_{i} \leq \frac{k_{i}}{2}$ implies $k_{i}-a_{i}>0$. Also observe that $F\left(\left.\rho_{j}\right|_{R_{i-1}}\right)$ and $F\left(\left.\rho_{j} \rho_{i}\right|_{R_{i-1}}\right)$ intersect transversely in $R_{i-1}$ with intersection $R_{i}$. If the codimensions $a_{i}$ and $k_{i}-a_{i}$ satisfy

$$
2 a_{i}+2\left(k_{i}-a_{i}\right) \leq \operatorname{dim}\left(R_{i-1}\right),
$$

then Corollary 4.3 would imply that $\mathrm{b}_{\text {odd }}\left(R_{i-1}\right)=0$. Since $R_{i-1}=F\left(\left\langle\rho_{1}, \ldots, \rho_{i-1}\right)\right.$, this would complete the proof.

To check the required bound on the codimensions, first note that Lemma 5.2 implies $\operatorname{dim}\left(R_{1}\right)>(\operatorname{dim} N) / \sqrt{2}$, hence $k_{1} \leq(\operatorname{dim} N) / 2$. Next, note that $\operatorname{dim}\left(R_{i-1}\right)=$ $(\operatorname{dim} N)-\left(k_{1}+\cdots+k_{i-1}\right)$, so the bound we wish to verify is equivalent to

$$
2 k_{i}+k_{i-1}+\cdots+k_{1} \leq \operatorname{dim} N .
$$

But $2 k_{h} \leq k_{h-1}$ for all $h$, so the left-hand side is at most $2 k_{1}$ and hence at most $\operatorname{dim} N$. This completes the proof of Lemma 5.8.

To summarize, we have shown in all five cases the existence of a submanifold $F \subseteq$ $P \subseteq N$ on which $T^{\prime}$ acts such that $\mathrm{b}_{\text {odd }}(P)=0$. As explained at the beginning of the proof, Conner's theorem implies $b_{\text {odd }}(F)=0$, so by repeating this argument for all components $F \subseteq M^{T}$, we have $\mathrm{b}_{\text {odd }}\left(M^{T}\right)=0$. By Berger's theorem, $M^{T}$ is nonempty, so $\chi(M)=\chi\left(M^{T}\right)>0$, as claimed.

\section{A corollary and a conjecture}

Our first point of discussion regards general Lie group actions. By examining the list of simple Lie groups, one easily shows that $(2 \operatorname{rank}(G))^{2} \geq \operatorname{dim}(G)$ for all compact, one-connected, simple Lie groups. The inequality persists for all compact Lie groups. In addition, $\operatorname{dim}\left(M^{n} / G\right) \leq n-d$ clearly implies $\operatorname{dim}(G) \geq d$. Hence, letting $I(M)$ denote the isometry group of $M$, we have the following corollary:

Corollary 6.1 Let $M^{n}$ be a closed Riemannian manifold with positive sectional curvature and $n \equiv 0 \bmod 4$. If $\operatorname{dim} I(M) \geq\left(4 \log _{2} n-4\right)^{2}$ or $\operatorname{dim} M / I(M) \leq$ $n-\left(4 \log _{2} n-4\right)^{2}$, then $\chi(M)>0$.

We remark that, for closed, even-dimensional manifolds with positive sectional curvature, it was shown in Püttmann and Searle [13] that $\chi\left(M^{n}\right)>0$ if $\operatorname{dim} M / I(M)<6$, and it follows from the main theorems in Wilking [18] that $\chi\left(M^{n}\right)>0$ if $\operatorname{dim} M / I(M) \leq \sqrt{n} / 18-1$ or $\operatorname{dim} I(M) \geq 2 n-6$.

To conclude, we state a conjecture that would improve the conclusion of the periodicity theorem. Recall that the periodicity theorem rested on Propositions 1.3 and 2.1, which 
we referred to as generalizations of Adem's theorem on singly generated cohomology rings. The conclusion of Adem's theorem when $p=2$ was improved by Adams after he developed the theory of secondary cohomology operations (see [1]). The analogous strengthening for odd primes is due to Liulevicius [11], and Shimada and Yamanoshita [15]. The result is the following:

Theorem 6.2 (Adams, Liulevicius, Shimada and Yamanoshita) Let $p$ be a prime, and let $M$ be a topological space. Assume $H^{*}\left(M ; \mathbb{Z}_{p}\right)$ is isomorphic to $\mathbb{Z}_{p}[x]$ or $\mathbb{Z}_{p}[x] / x^{q+1}$ with $p \leq q$. Set $k=\operatorname{deg}(x)$.

(1) If $p=2$, then $k \in\{1,2,4,8\}$. Moreover, $k=8$ only occurs when $q=2$.

(2) If $p>2$, then $k=2 \lambda$ for some $\lambda \mid p-1$.

Observe that singly generated cohomology rings are periodic in the sense of this paper. The corresponding strengthening in our case would be the following:

Conjecture 6.3 Let $p$ be a prime, and let $M$ be a topological space. Assume $x \in$ $H^{k}\left(M ; \mathbb{Z}_{p}\right)$ is nonzero and induces periodicity up to degree $p k$, and suppose $x$ has minimal degree among all such elements.

(1) If $p=2$, then $k \in\{1,2,4,8\}$. Moreover, if $x$ induces periodicity up to degree $3 k$, then $k \neq 8$.

(2) If $p>2$, then $k=2 \lambda$ for some $\lambda \mid p-1$.

We first note that, regarding the first statement, $\mathbb{S}^{k-1} \times \mathbb{S}^{k}$ is $k$-periodic but not $k^{\prime}$-periodic for any $k^{\prime}<k$, and $\mathbb{S}^{7} \times \mathrm{CaP}^{2}$ is 8 -periodic but not 4 -periodic. Hence one must assume periodicity up to degree $2 k$, respectively $3 k$.

Second, we wish to outline how a proof of this conjecture would imply that Theorem A holds in all even dimensions. First, one would use the conjecture to improve Proposition 1.3 to prove the following: If $M$ is a simply connected, closed manifold such that $H^{*}\left(M^{n} ; \mathbb{Z}_{2}\right)$ is $k$-periodic with $3 k \leq n$, then $M$ has the $\mathbb{Z}_{2}$-cohomology ring of $\mathbb{S}^{n}, \mathbb{C} \mathbb{P}^{n / 2}, \mathbb{H} \mathbb{P}^{n / 4}, \mathbb{H} \mathbb{P}^{(n-3) / 4} \times \mathbb{S}^{3}$ or $\mathbb{H} \mathbb{P}^{(n-2) / 4} \times \mathbb{S}^{2}$. Indeed, a proof of the $\mathbb{Z}_{2}-$ periodicity conjecture combined with Poincaré duality implies this when $n \not \equiv 2 \bmod 4$.

Suppose then that $n \equiv 2 \bmod 4$. We may assume without loss of generality that $H^{4}\left(M ; \mathbb{Z}_{2}\right) \cong \mathbb{Z}_{2}$ and that the generator $x$ has minimal degree among all elements inducing periodicity. It follows from Lemmas 1.2 and 1.4 that $\operatorname{Sq}^{1}\left(H^{3}\left(M ; \mathbb{Z}_{2}\right)\right)=0$, $\operatorname{Sq}^{1}\left(H^{7}\left(M ; \mathbb{Z}_{2}\right)\right)=0$ and $\operatorname{Sq}^{2}\left(H^{2}\left(M ; \mathbb{Z}_{2}\right)\right)=0$.

By periodicity and Poincaré duality, $H^{2}\left(M ; \mathbb{Z}_{2}\right) \cong \mathbb{Z}_{2}$. Let $z_{2} \in H^{2}\left(M ; \mathbb{Z}_{2}\right)$ be a generator. If $H^{3}\left(M ; \mathbb{Z}_{2}\right)=0$, it follows that $H^{*}\left(M ; \mathbb{Z}_{2}\right) \cong H^{*}\left(\mathbb{S}^{2} \times \mathbb{H} \mathbb{P}^{(n-2) / 4} ; \mathbb{Z}_{2}\right)$. 
To see that this is the case, suppose there exists a nonzero $u \in H^{3}\left(M ; \mathbb{Z}_{2}\right)$. Using Poincaré duality and periodicity again, we conclude the existence of a relation $u v=$ $x z$ for some $v \in H^{3}\left(M ; \mathbb{Z}_{2}\right)$. One can now use the Cartan formula to prove that $\mathrm{Sq}^{4}(u v)=0$ and $\mathrm{Sq}^{4}(x z)=x^{2} z \neq 0$, which is a contradiction.

Given this, the basic outline of our proof of Theorem A implies the result without the assumption that the dimension is divisible by four. In fact, the proof simplifies since one does not have to keep track of the divisibility of the codimensions. The optimal bound, as far as the proof is concerned, would be $r \geq \log _{2}(n)-2$.

\section{References}

[1] J F Adams, On the non-existence of elements of Hopf invariant one, Ann. of Math. 72 (1960) 20-104 MR0141119

[2] J Adem, The iteration of the Steenrod squares in algebraic topology, Proc. Nat. Acad. Sci. U. S. A. 38 (1952) 720-726 MR0050278

[3] M Berger, Trois remarques sur les variétés riemanniennes à courbure positive, $\mathrm{C}$. $\mathrm{R}$. Acad. Sci. Paris Sér. A-B 263 (1966) A76-A78 MR0199823

[4] R L Bishop, S I Goldberg, Some implications of the generalized Gauss-Bonnet theorem, Trans. Amer. Math. Soc. 112 (1964) 508-535 MR0163271

[5] S-s Chern, On curvature and characteristic classes of a Riemann manifold, Abh. Math. Sem. Univ. Hamburg 20 (1955) 117-126 MR0075647

[6] P E Conner, On the action of the circle group, Michigan Math. J. 4 (1957) 241-247 MR0096747

[7] O Dearricott, A 7-manifold with positive curvature, Duke Math. J. 158 (2011) 307-346 MR2805071

[8] K Grove, Developments around positive sectional curvature, from: "Surveys in differential geometry. Vol. XIII. Geometry, analysis, and algebraic geometry: forty years of the Journal of Differential Geometry", (H-D Cao, S-T Yau, editors), Surv. Differ. Geom. 13, International Press (2009) 117-133 MR2537084

[9] K Grove, L Verdiani, W Ziller, An exotic $T_{1} \mathbb{S}^{4}$ with positive curvature, Geom. Funct. Anal. 21 (2011) 499-524 MR2810857

[10] A Hatcher, Algebraic topology, Cambridge Univ. Press (2002) MR1867354

[11] A Liulevicius, The factorization of cyclic reduced powers by secondary cohomology operations, Mem. Amer. Math. Soc. No. 42 (1962) 112 MR0182001

[12] P Petersen, F Wilhelm, An exotic sphere with positive curvature arXiv: $0805.0812 \mathrm{v} 3$ 
[13] T Püttmann, C Searle, The Hopf conjecture for manifolds with low cohomogeneity or high symmetry rank, Proc. Amer. Math. Soc. 130 (2002) 163-166 MR1855634

[14] X Rong, X Su, The Hopf conjecture for manifolds with abelian group actions, Commun. Contemp. Math. 7 (2005) 121-136 MR2129791

[15] N Shimada, T Yamanoshita, On triviality of the mod $p$ Hopf invariant, Japan J. Math. 31 (1961) 1-25 MR0148060

[16] X Su, Y Wang, The Hopf conjecture for positively curved manifolds with discrete abelian group actions, Differential Geom. Appl. 26 (2008) 313-322 MR2421709

[17] B Wilking, Torus actions on manifolds of positive sectional curvature, Acta Math. 191 (2003) 259-297 MR2051400

[18] B Wilking, Positively curved manifolds with symmetry, Ann. of Math. 163 (2006) 607-668 MR2199227

[19] B Wilking, Nonnegatively and positively curved manifolds, from: "Surveys in differential geometry. Vol. XI", (J Cheeger, K Grove, editors), Surv. Differ. Geom. 11, International Press (2007) 25-62 MR2408263

[20] W Ziller, Examples of Riemannian manifolds with non-negative sectional curvature, from: "Surveys in differential geometry. Vol. XI", (J Cheeger, K Grove, editors), Surv. Differ. Geom. 11, International Press (2007) 63-102 MR2408264

Department of Mathematics, University of California, Santa Barbara Santa Barbara, CA 93106-3080, USA

kennard@math.ucsb.edu

http://www. math.ucsb.edu/ kennard

Proposed: John Lott

Seconded: Dmitri Burago, Steve Ferry
Received: 29 May 2012 Revised: 17 November 2012 